\title{
The first total synthesis of natural grenadamide $\uparrow$
}

\author{
Thomas D. Avery, Julie A. Culbert and Dennis K. Taylor*
}

Received 28th September 2005, Accepted 4th November 2005

First published as an Advance Article on the web 12th December 2005

DOI: 10.1039/b513774h

A concise, high yielding route to the naturally occurring enantiomer of grenadamide utilizing a

3,6-disubstituted 1,2-dioxine starting material is presented. The route allows for ease in synthesizing grenadamide derivatives varying at cyclopropyl carbons 2 and 3, with access to both enantiomers.

Evidence for phosphorus-assisted deprotonation of 1,2-dioxines is also discussed.

\section{Introduction}

Grenadamide is a naturally occurring cyclopropyl amide isolated along with debromogrenadadiene and grenadadiene by Gerwick and Sitachitta from marine cyanobacterium Lyngbya majuscula. ${ }^{1}$ Cascarillic acid is another related trans-cyclopropyl fatty acid isolated from the bark of Croton eluteria Benett, a West Indian tree-like shrub. ${ }^{2}$ These natural products are all based on a simple trans-substituted cyclopropyl fatty acid core, Fig. 1.

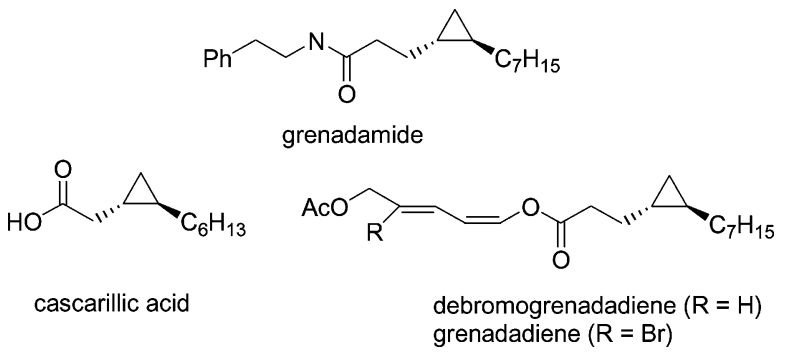

Fig. 1 Natural products containing related trans-cyclopropyl fatty acid cores.

Grenadamide exhibits modest activity in cannabinoid receptor binding activity assays and brine shrimp toxicity. ${ }^{1}$ In order to optimize the biological activity and deduce any structureactivity relationships, derivatives of grenadamide are required. Modification of the grenadamide structure at the amide linkage can be easily achieved through the carboxylic acid, with the more synthetically challenging motifs being at carbons 2 and 3 of the cyclopropyl ring.

Non-natural grenadamide has recently been synthesized by Baird et al. using an enantioselective enzymatic hydrolysis to generate the required chiral starting material. ${ }^{3}$ This synthesis allowed the absolute stereochemistry of natural grenadamide to be determined.

Discussed in this paper is the concise synthesis of (+)- and (-)grenadamide using the coupling of an intermediate cyclopropyl carboxylic acid with Evans' auxiliary as the method by which the enantiomers were resolved. Utilizing a diastereoselective cy-

Department of Chemistry, University of Adelaide, South Australia, 5005, Australia.E-mail: dennis.taylor@adelaide.edu.au

$\dagger$ Electronic supplementary information (ESI) available: Variable temperature NMR studies. See DOI: 10.1039/b513774h clopropanation sequence, trisubstituted cyclopropanes exhibiting the desired stereochemistry can be rapidly generated, Scheme $1 .^{4 a, b}$

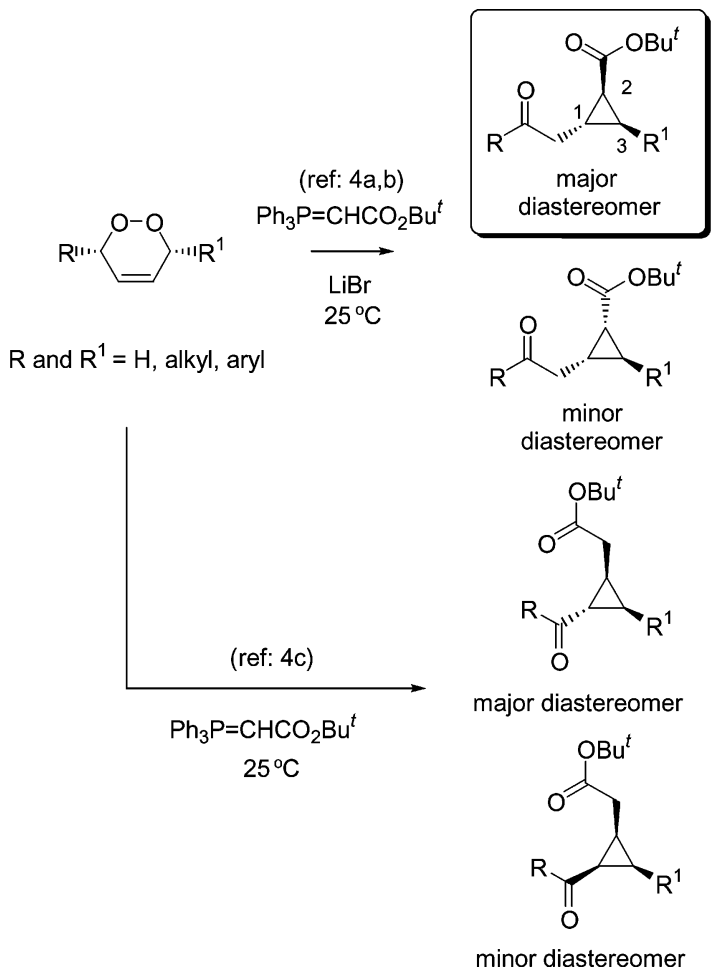

Scheme 1

Retrosynthetically we could achieve a rapid synthesis of grenadamide through the Arndt-Eistert homologation/amidation of carboxylic acid $\mathbf{2}$ which in turn could be obtained via hydrolysis of ester 3, Scheme 2. Ester 3 could be synthesized by Baeyer-Villiger oxidation of aromatic ketone 4 , which can be derived from cyclopropane $\mathbf{5 a}$ by ester hydrolysis and Barton decarboxylation. Finally, cyclopropanes of type 5a containing the required stereochemical relationships can be obtained via the aforementioned diastereoselective cyclopropanation protocol from 1,2-dioxine $\mathbf{6 a} \mathbf{a}^{4 a, b}$ This provides us with a route capable of introducing variation of cyclopropyl substituents at C2 and C3 and provides us with a suitable intermediate carboxylic acid with which to modify the amide linkage.

In order to accommodate the Baeyer-Villiger reaction in terms of reaction rate and functional group migration, an electron rich 


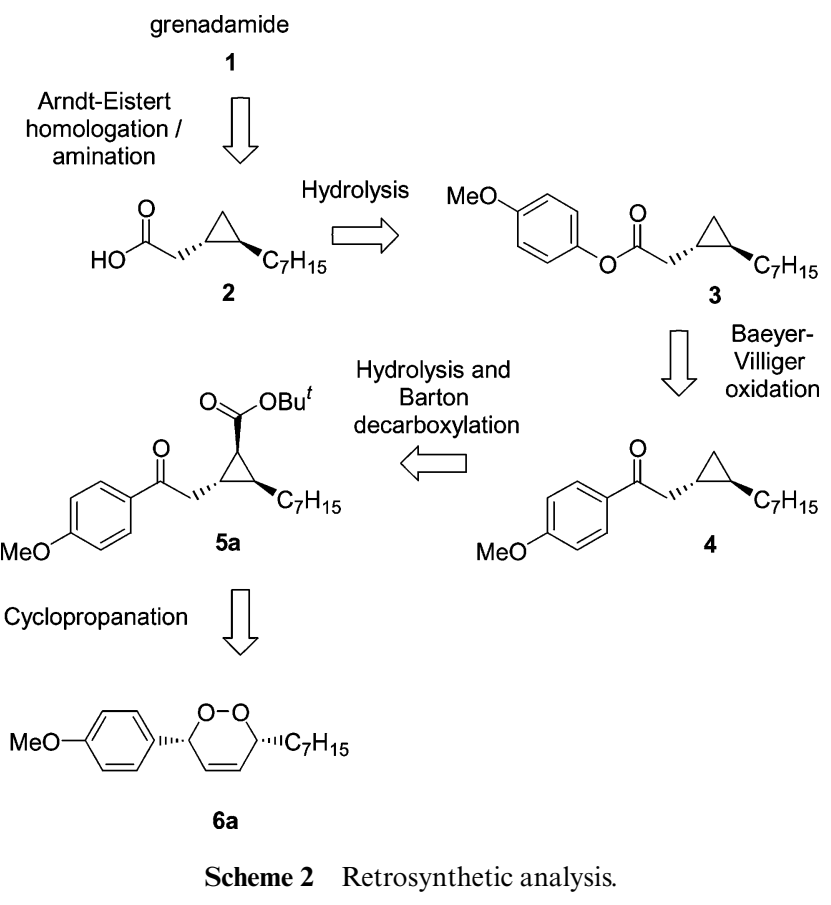

aromatic system is required. ${ }^{4 b}$ For ease of synthesis and functional group compatibility, the 4-methoxybenzene system was utilized.

1,2-Dioxines are effectively synthesized through photooxidation of their corresponding 1,3-dienes in the presence of a photosensitizer. Synthesis of the required 1,3-diene 8a was achieved in $77 \%$ yield via the Wittig coupling of 4methoxycinnamaldehyde $7 \mathbf{a}$ and octyltriphenylphosphonium iodide. Photolysis of 1,3-diene 8a in the presence of oxygen and rose bengal bis(triethylammonium) salt gave the desired 1,2-dioxine 6a in $37 \%$ yield, Scheme 3. 1,3-Diene 8a was reclaimed as the other major component of the photolysis and was able to be recycled.

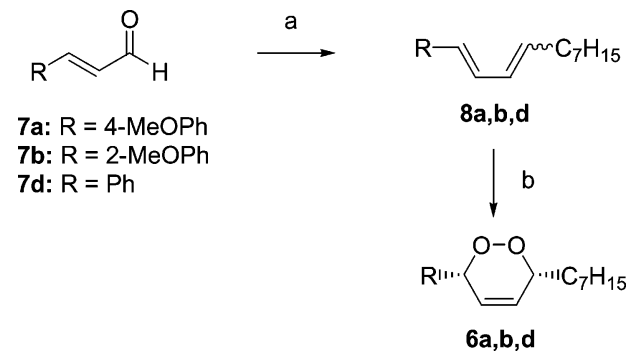

Scheme 3 a, Octyltriphenylphosphonium iodide (1.45 equiv.), $\mathrm{KO}^{t} \mathrm{Bu}$ (1.45 equiv.), THF, $0{ }^{\circ} \mathrm{C} \rightarrow \mathrm{rt}, 16 \mathrm{~h}$. b, $\mathrm{O}_{2}$, rose bengal bis(triethylammonium) salt (cat.), DCM, $h v, 5^{\circ} \mathrm{C}, 6 \mathrm{~h}$.

Reaction of the tert-butyl ester ylide with 1,2-dioxine 6a gave expected cyclopropane $\mathbf{5 a}$ in 55\% yield with isomer 9a making up the bulk of the remaining isolable reaction products, Table 1, entry 1 (Scheme 4).

Cyclopropane 5a was the expected and desired product, however, cyclopropane 9a was, unusually, the product resulting from 1,2-dioxine ring-opening through removal of the least acidic proton; that adjacent to the heptyl side-chain. This isomer had not previously been observed when subjecting 1,2-dioxine $\mathbf{6 c}$ to identical reaction conditions, Table 1 , entry $3 .^{4 a, b}$ Therefore, it appears that the presence of the extended alkyl chain is causing
Table 1 Optimization of cyclopropanation

\begin{tabular}{clll}
\hline Entry $^{a}$ & 1,2-Dioxine & Reaction time & $\begin{array}{l}\text { Isolated yield } \\
(\mathbf{5 : 9 : 1 0 : 1 1 ,} \%)\end{array}$ \\
\hline 1 & $\mathbf{6 a}$ & 15 days & $55: 20: 0: 0$ \\
2 & $\mathbf{6 b}$ & 30 days & $50: 22: 0: 8$ \\
$3^{b}$ & $\mathbf{6 c}$ & 4 days & $78: 0: 0: 0$ \\
4 & $\mathbf{6 d}$ & 7 days & $52: 18: 0: 0$ \\
$5^{c}$ & $\mathbf{6 b}$ & 2 days & $11: 5: 0: 0^{d}$ \\
$6^{e}$ & $\mathbf{6 b}$ & 30 days & $0: 0: 35: 58^{f}$ \\
$7^{g}$ & $\mathbf{6 b}$ & 30 days & $46: 24: 0: 9$ \\
$8^{h}$ & $\mathbf{6 b}$ & 31 days & $15: 6: 11: 24^{i}$ \\
$9^{j}$ & $\mathbf{6 b}$ & 32 days & $24: 15: 6: 13$ \\
$10^{k}$ & $\mathbf{6 b}$ & 2 days & $81: 0: 0: 0$ \\
$11^{l}$ & $\mathbf{6 b}$ & 1 day & $11: 11: 0: 0^{m}$ \\
$12^{k}$ & $\mathbf{6 a}$ & 2 days & $83: 0: 0: 0$
\end{tabular}

${ }^{a}$ Reactions were carried out using 1.1 equiv. $\mathrm{LiBr}$ and 1.4 equiv. $\mathrm{Ph}_{3} \mathrm{P}=\mathrm{CHCO}_{2}{ }^{t} \mathrm{Bu}$ in DCM at a concentration of $0.06 \mathrm{M}$ with respect to 1,2-dioxine unless otherwise stated. ${ }^{b}$ Reaction was carried out using 1.2 equiv. $\mathrm{Ph}_{3} \mathrm{P}=\mathrm{CHCO}_{2}{ }^{t} \mathrm{Bu}$ in $\mathrm{DCM}$ at a concentration of $0.2 \mathrm{M}$ with respect to 1,2-dioxine. ${ }^{c}$ Reaction carried out in $\mathrm{CHCl}_{3}$ at reflux. ${ }^{d}$ Major product was corresponding furan from cyclization and dehydration of cis$\gamma$-hydroxy enone intermediate. ${ }^{4 a}{ }^{e}$ Reaction carried out in the presence of 0.1 equiv. LiBr. ${ }^{f}$ Yields adjusted to account for $58 \%$ reclaimed starting material. ${ }^{g}$ Reaction carried out in the absence of light. ${ }^{h}$ Reaction photolyzed for $5 \mathrm{~h}$ each day for the first two days of reaction with a sun lamp. ${ }^{i}$ Yields adjusted to account for $47 \%$ reclaimed starting material. ${ }^{j}$ Reaction carried out in the presence of 1.1 equiv. of TEMPO. ${ }^{k}$ Reaction carried out in the presence of triethylamine. ${ }^{\prime}$ Reaction carried out in the presence of 0.1 equiv. of $\mathrm{Co}(\mathrm{II})$-salen. ${ }^{m} 24 \%$ bis(epoxide)s isolated. ${ }^{5}$

this alteration in reaction mechanism. This was confirmed by the synthesis of analogue $\mathbf{6 d}$, which showed formation of cyclopropane 9d when subjected to the reaction conditions, entry 4 .

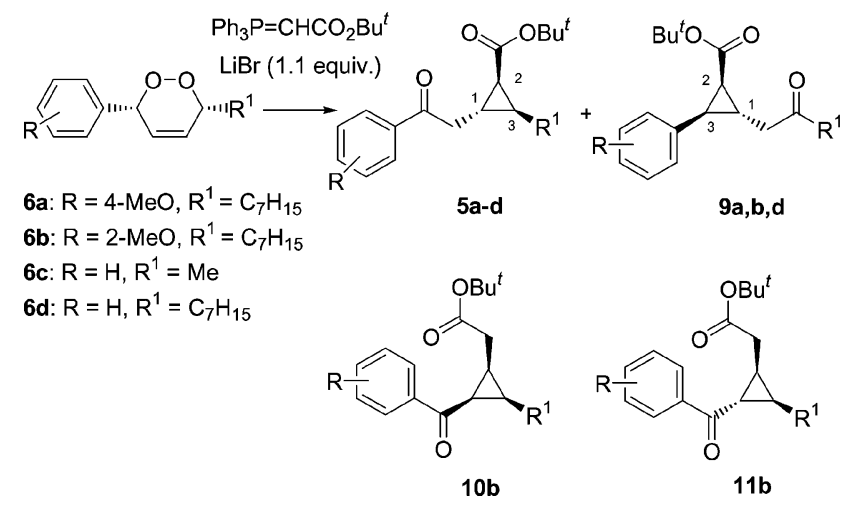

Scheme 4

The first step in cyclopropanation is removal of the most acidic proton adjacent to the $\mathrm{O}-\mathrm{O}$ linkage of the 1,2-dioxine, which induces rearrangement to give the $c i s-\gamma$-hydroxy enone 12, Scheme 5. The cis- $\gamma$-hydroxy enone undergoes Michael addition and concomitant $\mathrm{P}-\mathrm{O}$ bond formation with the stabilized phosphorus ylide giving an oxaphospholane intermediate ultimately leading to cyclopropane $\mathbf{5}^{4 b, c}$

It is this first step along the reaction pathway that is being inhibited by the presence of the extended alkyl chain, as we see no appreciable decomposition of 1,2-dioxine over these extended reaction times. If the rearrangement to $c i s-\gamma$-hydroxy enone was occurring rapidly we would expect to see $c i s$ - $\gamma$-hydroxy enone formation followed by 1,4-diketone formation through its base 


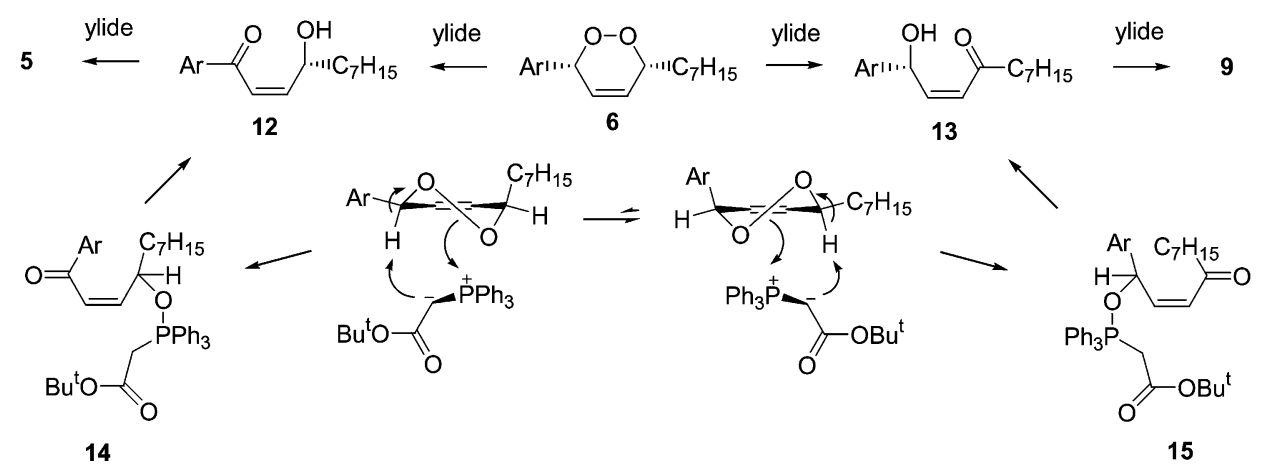

Scheme 5

catalyzed decomposition. ${ }^{4}$ This led us to propose that the extended alkyl chain is essentially fixing the conformation of the 1,2-dioxine so that the alkyl chain is pseudo-equatorial ${ }^{5}$ and that the removal of the proton adjacent the $\mathrm{O}-\mathrm{O}$ linkage is greatly assisted by the phosphorus of the ylide, Scheme 5. Indeed this is potentially the primary mechanism through which phosphorus stabilised ylides facilitate the transformation of 1,2-dioxines into cis $\gamma$-hydroxy enones. This mechanism allows for charge stabilization of the ylide, as well as proceeding through a very stable 6-membered transition state. In the case of 1,2-dioxine 6c, low temperature NMR spectroscopy has revealed that the Me substituent is predominantly pseudo-equatorial $(69: 31)$ at this temperature. ${ }^{5}$ Variable temperature ${ }^{1} \mathrm{H}$ NMR carried out on $\mathbf{6 b}$ demonstrated that when $\mathrm{R}^{1}$ is very large then the conformation is essentially fixed with $\mathrm{R}^{1}$ pseudo-equatorial (9:1), as only a single conformer was observed at $-91^{\circ} \mathrm{C}$. This strongly supports the notion that the conformer with the extended alkyl chain pseudo axial, is present at a considerably lower percentage of the population than its pseudo equatorial counterpart at ambient temperature. Due to the low population the reaction rate through $\mathbf{1 4}$ is extended to a point where the generation of cyclopropane $\mathbf{9 a}, \mathbf{b}$ and $\mathbf{d}$, through intermediate $\mathbf{1 5}$, competes on the timescale of the reaction. In concert with this hypothesis is the observation that with increasing electron density of the aromatic ring and steric bulk ( $6 \mathbf{d} \rightarrow \mathbf{a} \rightarrow \mathbf{b})$ we observe an increase in reaction time due to decreased acidity of the proton $\alpha$ to the aromatic ring and as expected a corresponding increase in the percent yield of cyclopropane $\mathbf{9}$, Table 1 .

1,2-Dioxine $\mathbf{6 b}$, which was also investigated, showed an identical product profile to $\mathbf{6 a}$ and $\mathbf{6 d}$ (Table 1, entries 1 and 4), with the exception that cyclopropane isomer 11b was observed, Table 1, entry 2 .

We thus chose to investigate the reaction of $\mathbf{6 b}$ in order to optimize formation of cyclopropane $\mathbf{5 b}$. Refluxing in chloroform resulted in a decreased reaction time but poor yield, entry 5 . Reduction of $\mathrm{LiBr}$ to 0.1 equivalents resulted in reversion to the alternate cyclopropane reaction pathway, entry 6 , Table 1 , see Scheme $1 .^{4 a}$ To investigate whether light played a part in the ringopening, through photo-induced peroxide bond cleavage, to give cyclopropane isomer $\mathbf{9 b}$, the reaction was carried out in both the presence and absence of all light. In the absence of light an essentially identical product profile was observed, compare entries 7 and 2, Table 1. However on photolyzing the reaction for 5 hours on each of the first two days of the reaction, we observed partial reversion to the alternate cyclopropanation pathway, entry 8 , indicating that exposure of these reactions to light was not causal in the formation of $\mathbf{9}$. The addition of TEMPO to the reaction mixture, entry 9, allowed investigation of possible radical cleavage of the peroxide linkage, however, the ratio of 5:9 was identical to that in entry 2, 7:3 respectively. Due to the poor removal of the most acidic proton by the ylide, addition of triethylamine, which is known to rapidly ring-open 1,2-dioxines to their corresponding cis- $\gamma$-hydroxy enones via removal of the most acidic proton, was added to the reaction, entry 10 . This resulted in only isomer $\mathbf{5}$ being generated, indicating that cis- $\gamma$-hydroxy enone $\mathbf{1 2}$ is formed exclusively, giving $81 \%$ isolated yield. The rapid timeframe of the reaction indicates that rearrangement to $c i s-\gamma$-hydroxy enone is the rate determining step in the reaction.

The Taylor group has investigated the ring-opening of unsymmetrical 1,2-dioxines using Co(II)-salen catalyst. ${ }^{5}$ However utilization of $\mathrm{Co}(\mathrm{II})$-salen to ring-open 1,2-dioxine $\mathbf{6 b}$ resulted in a $1: 1$ ratio of cyclopropanes $\mathbf{5 b}$ and $\mathbf{9 b}$ in poor yield, entry 11. This observation further supports Co(II) rearrangement of cis- $\gamma$-hydroxy enones is not solely dependent on 1,2-dioxine conformation. ${ }^{5}$

Using the optimized conditions cyclopropane 5a could now be generated in $83 \%$ isolated yield, entry 12 , Table 1 . With the desired cyclopropane in hand the tert-butyl ester was hydrolyzed cleanly in the presence of $99 \%$ formic acid to give acid $16,{ }^{6}$ in $89 \%$ yield, which was subsequently decarboxylated using the Barton protocol in $86 \%$ yield. ${ }^{7}$ Baeyer-Villiger oxidation of the resultant ketone 4 using MCPBA proceeded with excellent selectivity to give phenol ester 3 in $91 \%$ yield, Scheme $6 .^{4 b}$

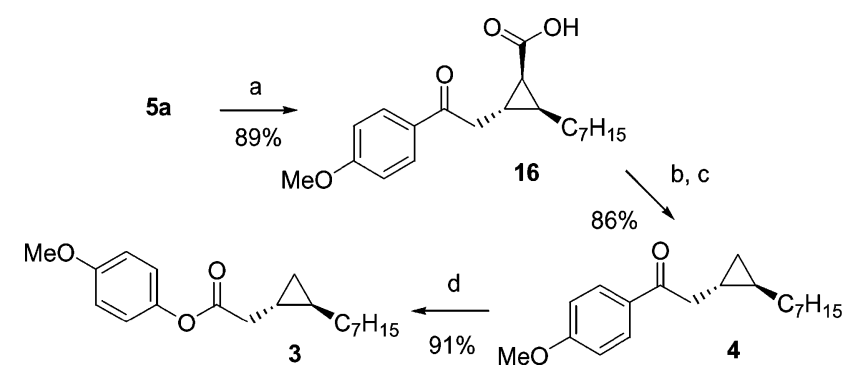

Scheme 6 a, 99\% Formic acid (excess), rt, 3 h. b, DCC (1 equiv.), 2-mercaptopyridine $N$-oxide (1 equiv.), DCM, $0{ }^{\circ} \mathrm{C}, 3$ h. c, ${ }^{t} \mathrm{BuSH}$ (2 equiv.), benzene, $h v, \mathrm{rt}, 2 \mathrm{~h}$. d, MCPBA 70\% (2 equiv.), DCM, rt, 2 days. 
Due to purification difficulties phenol ester $\mathbf{3}$ was transesterified to furnish methyl ester $\mathbf{1 7}$ in $93 \%$ yield, which was subsequently hydrolyzed to give cyclopropyl fatty acid 2 in $93 \%$ yield. ${ }^{4 b}$ ArndtEistert homologation/amidation ${ }^{8}$ of acid 2 proceeded cleanly to afford racemic grenadamide in $83 \%$ yield, through acid chloride ${ }^{9}$ and diazoketone ${ }^{10}$ intermediates, Scheme 7.

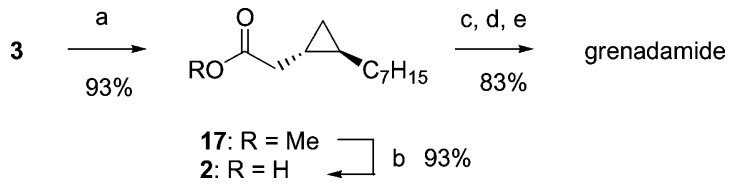

Scheme 7 a, $\mathrm{MeOH}$ (excess), conc. $\mathrm{H}_{2} \mathrm{SO}_{4}$ (cat.) reflux, 16 h. b, $2 \mathrm{~N} \mathrm{KOH}$ (1.4 equiv.), $\mathrm{MeOH}-\mathrm{H}_{2} \mathrm{O}$ (4:1), rt, 6 h. c, Oxalyl chloride (3 equiv.), DMF (cat.), DCM, $0{ }^{\circ} \mathrm{C}(2 \mathrm{~h}) \rightarrow \mathrm{rt}(2 \mathrm{~h}) . \mathrm{d}, \mathrm{CH}_{2} \mathrm{~N}_{2}$ (1.5 equiv.), triethylamine ( 1.5 equiv.), diethyl ether, $0{ }^{\circ} \mathrm{C}, 0.5 \mathrm{~h}$. e, $\mathrm{PhCO}_{2} \mathrm{Ag}$ (1.1 equiv.), phenethylamine (4 equiv.), THF, triethylamine, $-15^{\circ} \mathrm{C} \rightarrow \mathrm{rt}, 16 \mathrm{~h}$.

To obtain the two enantiomers of grenadamide, cyclopropyl fatty acid 2 was resolved by coupling it with Evans' auxiliary and chromatographically separating the diastereomers $\mathbf{1 8}$ and $\mathbf{1 9}$ generated, Scheme 8. ${ }^{9}$ The auxiliary was then cleaved and the enantiomerically pure fatty acids were subjected to the ArndtEistert protocol to give (-)- and (+)-grenadamide from (-)- and $(+)-2$ respectively. Resolution in this manner using carboxylic acid 16 was not feasible due to incompatibility of the ketone moiety with the conditions required to remove the Evans' auxiliary.

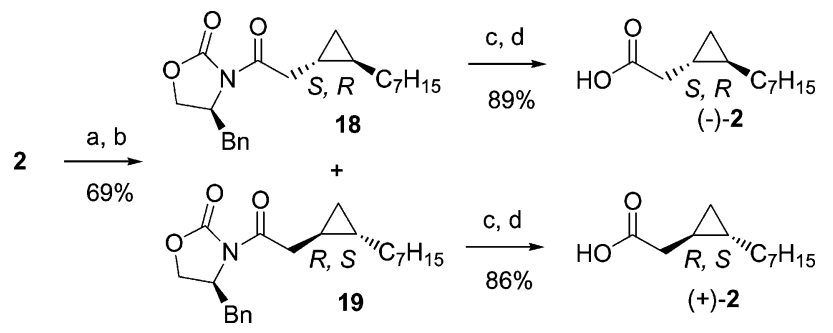

Scheme 8 a, Oxalyl chloride ( 3 equiv.), DMF (cat.), DCM, $0{ }^{\circ} \mathrm{C}(2 \mathrm{~h}) \rightarrow$ rt (2 h) b, (S)-(-)-4-Benzyl-2-oxazolidinone (1 equiv.), $n \mathrm{BuLi}$ (1 equiv.), THF, $-78{ }^{\circ} \mathrm{C}, 4$ h. c, Chromatographic separation. d, LiOH (4 equiv.), $\mathrm{H}_{2} \mathrm{O}_{2} 30 \%$ (8 equiv.), $\mathrm{H}_{2} \mathrm{O}$, THF, $0{ }^{\circ} \mathrm{C}, 0.5 \mathrm{~h}$.

Grenadamide synthesized from 19 gave an optical rotation $[a]_{\mathrm{D}}^{20.5}+12.0\left(\mathrm{CHCl}_{3}, c=0.005\right)$ corresponding to that synthesized by Baird et al. $\left([\alpha]_{\mathrm{D}}^{22}+12.6\left(\mathrm{CHCl}_{3}, c=0.84\right)\right)^{3}$ with the grenadamide synthesized from 18 exhibiting an equal and opposite rotation $[a]_{\mathrm{D}}^{20.5}-12.0\left(\mathrm{CHCl}_{3}, c=0.005\right)$ which corresponded to that of the natural grenadamide isolated by Gerwick and Sitachitta $\left([a]_{\mathrm{D}}-11.0\left(\mathrm{CHCl}_{3}, c=0.1\right)\right){ }^{1}$

The route to grenadamide reported herein lends itself to the easy synthesis of grenadamide derivatives. Variation at carbon 3 of the cyclopropyl ring can be achieved through the use of different phosphonium salts in the generation of the 1,3-diene for photooxidation. Modification of carbon 2 of the cyclopropyl ring can be achieved by manipulation of the ester functional group, which is at this stage redundant in the synthesis of grenadamide. This synthesis also lends itself to the synthesis of cascarillic acid as cascarillic acid is only 1 carbon shorter in the alkyl side chain than cyclopropyl acid 2.

\section{Experimental}

\section{General}

Solvents were dried by appropriate methods wherever needed. Thin-layer chromatography (TLC) used aluminium sheets coated with silica gel $60 \mathrm{~F}_{254}(40 \times 80 \mathrm{~mm})$ and visualized under $254 \mathrm{~nm}$ light, or developed in vanillin or permanganate dip. Column chromatography was conducted using silica gel 60 of particle size $0.040-0.063 \mathrm{~mm}$. Melting points are uncorrected. ${ }^{1} \mathrm{H}$ NMR and ${ }^{13} \mathrm{C}$ NMR spectra were recorded in $\mathrm{CDCl}_{3}$ solution at either $300 \mathrm{MHz}$ or $600 \mathrm{MHz}$ with TMS (0 ppm) and $\mathrm{CDCl}_{3}(77.0 \mathrm{ppm})$ as internal standards. All yields reported refer to isolated material judged to be homogeneous by TLC and NMR spectroscopy.

\section{General procedure for the synthesis of 1,3-dienes 8}

To a suspension of octyltriphenylphosphonium iodide (29.2 g, $58.1 \mathrm{mmol})$ in anhydrous THF $(10 \mathrm{ml}$ per $\mathrm{g}$ of phosphonium salt) at ambient temperature and under a nitrogen atmosphere was added potassium tert-butoxide (1.2 equiv.). After stirring for 15 minutes the orange solution was cooled to $0{ }^{\circ} \mathrm{C}$ and a solution of aldehyde 7a,b,d (1 equiv.) in anhydrous THF (10 ml per $\mathrm{g}$ of 7) was added dropwise. The solution was stirred for 16 hours at ambient temperature, diluted with hexanes and filtered through silica gel. The silica was washed with 1:19 ethyl acetate-hexanes. The filtrate was concentrated in vacuo and the resulting residue purified by column chromatography using 1:99 ethyl acetatehexanes as eluent to yield 1,3-dienes 8a,b,d.

$(1 E, 3 E)$ and $(1 E, 3 Z)-1-(4-M e t h o x y p h e n y l)-1,3-$ undecadiene 8a. $R_{\mathrm{f}} 0.39$; $(7.99 \mathrm{~g}, 77 \%)$, in a ratio of $95: 5,1 E, 3 E: 1 E, 3 Z$ as a colourless oil. $E, E$ isomer: $\delta_{\mathrm{H}}\left(300 \mathrm{MHz} ; \mathrm{CDCl}_{3} ; \mathrm{Me}_{4} \mathrm{Si}\right) 0.85-$ $0.90(3 \mathrm{H}, \mathrm{m}, \mathrm{Me}), 1.26-1.44\left(10 \mathrm{H}, \mathrm{m}, \mathrm{CH}_{2}\right), 2.23-2.32(2 \mathrm{H}, \mathrm{m}$, $\left.\mathrm{CH}_{2} \mathrm{CH}=\mathrm{CH}\right), 3.80(3 \mathrm{H}, \mathrm{s}, \mathrm{OMe}), 5.49(1 \mathrm{H}, \mathrm{ddd}, J 10.8,6.0$ and $6.0 \mathrm{~Hz}, \mathrm{CH}=\mathrm{CH}), 6.13(1 \mathrm{H}, \mathrm{dd}, J 10.8$ and $10.8 \mathrm{~Hz}, \mathrm{CH}=\mathrm{CH})$, $6.47(1 \mathrm{H}, \mathrm{d}, J 15.3 \mathrm{~Hz}, \mathrm{CH}=\mathrm{CH}), 6.82-6.89(2 \mathrm{H}, \mathrm{m}, \mathrm{Ph}), 6.93$ $(1 \mathrm{H}$, ddd, $J$ 15.3, 10.8 and $0.9 \mathrm{~Hz}, \mathrm{CH}=\mathrm{CH}), 7.32-7.36(2 \mathrm{H}, \mathrm{m}$, $\mathrm{Ph})$.

$(1 E, 3 E)$ and $(1 E, 3 Z)-1-(2-M e t h o x y p h e n y l)-1,3-$ undecadiene $8 \mathrm{~b}$. $R_{\mathrm{f}} 0.40 ;(11.45 \mathrm{~g}, 90 \%)$, in a ratio of $85: 15,1 E, 3 E: 1 E, 3 Z$ as a colourless oil. $E, E$ isomer: $\delta_{\mathrm{H}}\left(300 \mathrm{MHz} ; \mathrm{CDCl}_{3} ; \mathrm{Me}_{4} \mathrm{Si}\right) 0.86$ $0.91(3 \mathrm{H}, \mathrm{m}, \mathrm{Me}), 1.25-1.47\left(10 \mathrm{H}, \mathrm{m}, 5 \times \mathrm{CH}_{2}\right), 2.28(2 \mathrm{H}, \mathrm{q}, J$ $\left.6.6 \mathrm{~Hz}, \mathrm{CH}_{2}\right), 3.90(3 \mathrm{H}, \mathrm{s}, \mathrm{OMe}), 5.50(1 \mathrm{H}, \mathrm{dt}, J 10.8$ and $7.8 \mathrm{~Hz}$, $\mathrm{CH}=\mathrm{CH}), 6.19(1 \mathrm{H}, \mathrm{dd}, J 10.8$ and $10.8 \mathrm{~Hz}, \mathrm{CH}=\mathrm{CH}), 6.77-6.89$ $(2 \mathrm{H}, \mathrm{m}, \mathrm{CH}=\mathrm{CH}$ and $\mathrm{Ph}), 6.90-6.96(1 \mathrm{H}, \mathrm{m}, \mathrm{Ph}), 7.09(1 \mathrm{H}, \mathrm{dd}$, $J 15.6$ and $10.8 \mathrm{~Hz}, \mathrm{CH}=\mathrm{CH}), 7.17-7.23(1 \mathrm{H}, \mathrm{m}, \mathrm{Ph}), 7.48-7.51$ (1H, m, Ph).

$(1 E, 3 E)$ and $(1 E, 3 Z)-1-P h e n y l-1,3-$ undecadiene $8 \mathrm{~d} . \quad R_{\mathrm{f}} 0.55$; $(6.68 \mathrm{~g}, 67 \%)$, in a ratio of $20: 1,1 E, 3 Z: 1 E, 3 E$ as a colourless oil. $^{11}$

\section{General procedure for the synthesis of 1,2-dioxines 6}

A solution of 1,3-dienes $\mathbf{8 a , b , d}$ (1 equiv.) in anhydrous dichloromethane $(25 \mathrm{ml}$ per $\mathrm{g}$ of $\mathbf{8})$ was irradiated with light from $3 \times$ $500 \mathrm{~W}$ tungsten-halogen lamps in the presence of rose bengal bis(triethylammonium) salt (0.01 equiv.) and oxygen bubbled through the solution for 6 hours. The solution was concentrated and the resulting residue purified by column chromatography 
using 1:19 ethyl acetate-hexanes as eluent to yield 1,2-dioxine 6a,b,d.

4-(6-Heptyl-3,6-dihydro-1,2-dioxin-3-yl)phenyl methyl ether 6a. $R_{\mathrm{f}} 0.31 ;(5.65 \mathrm{~g}, 37 \%)$ as a colourless oil $v_{\max }($ film $) / \mathrm{cm}^{-1} 1689,1681$, $1612,1586,1513$ and $1465 ; \delta_{\mathrm{H}}\left(300 \mathrm{MHz} ; \mathrm{CDCl}_{3} ; \mathrm{Me}_{4} \mathrm{Si}\right) 0.87(3 \mathrm{H}$, $\mathrm{t}, J 6.6 \mathrm{~Hz}, \mathrm{Me}), 1.22-1.54\left(10 \mathrm{H}, \mathrm{m}, \mathrm{CH}_{2}\right), 1.56-1.65(1 \mathrm{H}, \mathrm{m}$, $\left.\mathrm{CH}_{2}\right), 1.73-1.80\left(1 \mathrm{H}, \mathrm{m}, \mathrm{CH}_{2}\right), 3.80(3 \mathrm{H}, \mathrm{s}, \mathrm{OMe}), 4.49-4.56(1 \mathrm{H}$, $\left.\mathrm{m}, \mathrm{C}_{7} \mathrm{H}_{15} \mathrm{CHCH}=\mathrm{CH}\right), 5.45-5.48(1 \mathrm{H}, \mathrm{m}, \mathrm{PhCHCH}=\mathrm{CH}), 6.00$ $6.13(2 \mathrm{H}, \mathrm{m}, \mathrm{CH}=\mathrm{CH}), 6.86-6.91(2 \mathrm{H}, \mathrm{m}, \mathrm{Ph}), 7.28-7.33(2 \mathrm{H}, \mathrm{m}$, $\mathrm{Ph}) ; \delta_{\mathrm{C}}\left(75 \mathrm{MHz} ; \mathrm{CDCl}_{3} ; \mathrm{Me}_{4} \mathrm{Si}\right)$ 14.0, 22.6, 25.5, 29.1, 29.5, 31.7, $33.1,55.2,78.4,79.8,113.8,126.5,128.8,129.7,130.1,160.0 ; \mathrm{m} / \mathrm{z}$ (EI) $290\left(\mathrm{M}^{+}, 16 \%\right), 272(53), 258$ (100), 187 (9), 173 (24), 134 (22); HRMS (ESI) $\mathrm{C}_{18} \mathrm{H}_{26} \mathrm{O}_{3}+\mathrm{Na}$ requires 313.1780, found 313.1776.

3-Heptyl-6-(2-methoxyphenyl)-3,6-dihydro-1,2-dioxine $6 \mathrm{~b} . \quad R_{\mathrm{f}}$ 0.39; $(5.98 \mathrm{~g}, 39 \%)$ as a colourless oil $v_{\max }($ film $) / \mathrm{cm}^{-1} 1603,1589$, 1493, 1464, 1248, 1030; $\delta_{\mathrm{H}}\left(300 \mathrm{MHz} ; \mathrm{CDCl}_{3} ; \mathrm{Me}_{4} \mathrm{Si}\right) 0.87(3 \mathrm{H}$, t, $J 7.2 \mathrm{~Hz}, \mathrm{Me}), 1.22-1.76\left(12 \mathrm{H}, \mathrm{m}, 6 \times \mathrm{CH}_{2}\right), 3.84(3 \mathrm{H}, \mathrm{s}$, $\mathrm{OMe}), 4.57-4.63\left(1 \mathrm{H}, \mathrm{m}, \mathrm{C}_{7} \mathrm{H}_{15} \mathrm{CHCH}=\mathrm{CH}\right), 5.94-5.97(1 \mathrm{H}, \mathrm{m}$, $\mathrm{PhCHCH}=\mathrm{CH}), 6.01-6.10(2 \mathrm{H}, \mathrm{m}, \mathrm{CH}=\mathrm{CH}), 6.87-6.97(2 \mathrm{H}, \mathrm{m}$, $\mathrm{Ph}), 7.27-7.33$ (1H, m, Ph), 7.37-7.40 (1H, m, Ph); $\delta_{\mathrm{C}}(75 \mathrm{MHz}$ $\mathrm{CDCl}_{3} ; \mathrm{Me}_{4} \mathrm{Si}$ ) 14.0, 22.6, 25.4, 29.1, 29.5, 31.8, 32.9, 55.5, 74.1, $78.3,110.6,120.3,126.2,126.4,128.6,129.2,129.7,157.3 ; \mathrm{m} / \mathrm{z}$ (EI) $290\left(\mathrm{M}^{+}, 25 \%\right), 272(31), 258(91), 187$ (79), 173 (37), 159 (40), 135 (100), 121 (67), 105 (16), 77 (61), 60 (67), 43 (62); HRMS (ESI) $\mathrm{C}_{18} \mathrm{H}_{26} \mathrm{O}_{3}+\mathrm{Na}$ requires 313.1780, found 313.1772.

3-Heptyl-6-phenyl-3,6-dihydro-1,2-dioxine 6d. $\quad R_{\mathrm{f}} 0.38$; (2.21 g, $59 \%$ ) as a colourless oil $v_{\max }($ film $) / \mathrm{cm}^{-1} 1682,1494,1455,1067$; $\delta_{\mathrm{H}}\left(200 \mathrm{MHz} ; \mathrm{CDCl}_{3} ; \mathrm{Me}_{4} \mathrm{Si}\right) 0.88(3 \mathrm{H}, \mathrm{t}, J 6.8 \mathrm{~Hz}, \mathrm{Me}), 1.20$ $1.80\left(12 \mathrm{H}, \mathrm{m}, 6 \times \mathrm{CH}_{2}\right), 4.53-4.58\left(1 \mathrm{H}, \mathrm{m}, \mathrm{C}_{7} \mathrm{H}_{15} \mathrm{CHCH}=\mathrm{CH}\right)$, 5.49-5.53 (1H, m, PhCHCH=CH), 6.02-6.16 (2H, m, CH=CH), 7.33-7.42 (5H, m, Ph); $\delta_{\mathrm{C}}\left(50 \mathrm{MHz} ; \mathrm{CDCl}_{3} ; \mathrm{Me}_{4} \mathrm{Si}\right)$ 14.0, 22.6, 25.5, 29.2, 29.5, 31.8, 33.1, 78.5, 80.2, 126.3, 128.5, 128.6, 128.7, 128.9, 137.8; m/z (EI) 260 (M+, 10\%), 228 (100), 143 (46), 129 (95), 105 (40); HRMS (ESI) $\mathrm{C}_{17} \mathrm{H}_{24} \mathrm{O}_{2}$ requires 260.1776, found 260.1765. Anal. Calcd for $\mathrm{C}_{17} \mathrm{H}_{24} \mathrm{O}_{2}$ requires $\mathrm{C}, 78.42 ; \mathrm{H}, 9.29$ : found $\mathrm{C}$, $78.12 ; \mathrm{H}, 9.34 \%$.

\section{General procedure for the synthesis of cyclopropanes from 6}

To a solution of 1,2-dioxine $\mathbf{6 a , b , \mathbf { d }}$ (1 equiv.) in anhydrous dichloromethane $(53 \mathrm{ml}$ per $\mathrm{g}$ of $\mathbf{6})$ was added lithium bromide (1.1 equiv.) and (tert-butoxycarbonylmethylene)triphenylphosphorane (1.4 equiv.). The mixture was stirred at ambient temperature for 15-30 days (Table 1). The solution was concentrated and the resulting residue purified by column chromatography using 3:17 ethyl acetate-hexanes as eluent to give cyclopropanes $\mathbf{5 a , b , d}$ and 9a,b,d.

( \pm -tert-Butyl [(1S,2R,3R)-2-heptyl-3-[2-(4-methoxyphenyl)-2oxoethyl]cyclopropane-1-carboxylate $5 \mathrm{a}$ and $( \pm)$-tert-butyl $[(1 R$, 2S,3S)-2-heptyl-3-(4-methoxybenzoyl)cyclopropyl]acetate 9a. Cyclopropane 5a: $R_{\mathrm{f}} \quad 0.43 ; \quad(4.83 \mathrm{~g}, \quad 55 \%)$ as a colourless oil $v_{\max }($ film $) / \mathrm{cm}^{-1} 1722,1715,1682,1602,1576$ and 1511; $\delta_{\mathrm{H}}\left(600 \mathrm{MHz} ; \mathrm{CDCl}_{3} ; \mathrm{Me}_{4} \mathrm{Si}\right) 0.86(3 \mathrm{H}, \mathrm{t}, J 6.6 \mathrm{~Hz}, \mathrm{Me}), 1.09-1.14$ $(1 \mathrm{H}, \mathrm{m}, \mathrm{C}(3) \mathrm{H}), 1.23-1.34\left(10 \mathrm{H}, \mathrm{m}, \mathrm{CH}_{2}\right), 1.44\left(9 \mathrm{H}, \mathrm{s},{ }^{t} \mathrm{Bu}\right), 1.50$ $(1 \mathrm{H}, \mathrm{dd}, J 9.0$ and $4.8 \mathrm{~Hz}, \mathrm{C}(2) \mathrm{H}), 1.53-1.67\left(3 \mathrm{H}, \mathrm{m}, \mathrm{CH}_{2} \mathrm{C}(3) \mathrm{H}\right.$ and $\mathrm{C}(1) H), 2.65\left(1 \mathrm{H}\right.$, dd, $J 16.2$ and $\left.8.4 \mathrm{~Hz}, \mathrm{C}(1) \mathrm{HCH}_{2}\right), 3.14$ $\left(1 \mathrm{H}, \mathrm{dd}, J 16.2\right.$ and $\left.6.0 \mathrm{~Hz}, \mathrm{C}(1) \mathrm{HCH}_{2}\right), 3.87(3 \mathrm{H}, \mathrm{s}, \mathrm{OMe}), 6.92-$
$6.94(2 \mathrm{H}, \mathrm{m}, \mathrm{Ph}), 7.89-7.91(2 \mathrm{H}, \mathrm{m}, \mathrm{Ph}) ; \delta_{\mathrm{C}}\left(75 \mathrm{MHz} ; \mathrm{CDCl}_{3}\right.$; $\left.\mathrm{Me}_{4} \mathrm{Si}\right)$ 14.0, 22.58, 22.61, 26.3, 26.7, 28.2, 29.1, 29.27, 29.32, 29.5, $31.8,41.9,55.4,80.1,113.8,129.8,130.4,163.5,171.4,197.2 ; \mathrm{m} / \mathrm{z}$ (EI) $388\left(\mathrm{M}^{+}, 40 \%\right), 332$ (33), 330 (36), 314 (100), 272 (38), 186 (7), 149 (7), 134 (55), 56 (29), 41 (18); HRMS (ESI) $\mathrm{C}_{24} \mathrm{H}_{36} \mathrm{O}_{4}+$ $\mathrm{Na}$ requires 411.2511, found 411.2513. Cyclopropane 9a: $R_{\mathrm{f}} 0.36$; $(1.76 \mathrm{~g}, 20 \%)$ as a colourless oil $v_{\max }($ film $) / \mathrm{cm}^{-1} 1714,1614,1515$, 1365,$1247 ; \delta_{\mathrm{H}}\left(600 \mathrm{MHz} ; \mathrm{CDCl}_{3} ; \mathrm{Me}_{4} \mathrm{Si}\right) 0.87(3 \mathrm{H}, \mathrm{t}, J 7.2 \mathrm{~Hz}$, $\mathrm{Me}), 1.17\left(9 \mathrm{H}, \mathrm{s},{ }^{t} \mathrm{Bu}\right), 1.22-1.31\left(8 \mathrm{H}, \mathrm{m}, 4 \times \mathrm{CH}_{2}\right), 1.57-1.62$ $\left(2 \mathrm{H}, \mathrm{m}, \mathrm{CH}_{2}\right), 1.76(1 \mathrm{H}, \mathrm{dd}, J 9.6$ and $5.4 \mathrm{~Hz}, \mathrm{C}(3) \mathrm{H}), 2.21(1 \mathrm{H}$, dddd, $J$ 8.4, 7.2, 5.4 and $5.4 \mathrm{~Hz}, \mathrm{C}(1) \mathrm{H}), 2.29(1 \mathrm{H}$, dd, $J 9.6$ and $7.2 \mathrm{~Hz}, \mathrm{C}(2) \mathrm{H}), 2.33\left(1 \mathrm{H}, \mathrm{dd}, J 16.8\right.$ and $\left.8.4 \mathrm{~Hz}, \mathrm{C}(1) \mathrm{HCH}_{2} \mathrm{C}(\mathrm{O})\right)$, 2.42-2.50 (2H, m, $\left.\mathrm{CH}_{2} \mathrm{C}(\mathrm{O})\right), 2.75(1 \mathrm{H}, \mathrm{dd}, J 16.8$ and $5.4 \mathrm{~Hz}$, $\left.\mathrm{C}(1) \mathrm{HCH}_{2} \mathrm{C}(\mathrm{O})\right), 3.77$ (3H, s, OMe), 6.79-6.81 (2H, m, Ph), 7.23$7.25(2 \mathrm{H}, \mathrm{m}, \mathrm{Ph}) ; \delta_{\mathrm{C}}\left(150 \mathrm{MHz} ; \mathrm{CDCl}_{3} ; \mathrm{Me}_{4} \mathrm{Si}\right)$ 14.0, 19.8, 22.6, 23.7, 27.8, 28.8, 29.1, 29.2, 31.6, 31.7, 42.6, 45.9, 55.3, 80.3, 113.4, $128.4,130.5,158.4,169.6,209.5 ; \mathrm{m} / z$ (EI) $388\left(\mathrm{M}^{+}, 15 \%\right), 332(19)$, 205 (44), 190 (39), 127(20), 67 (100); HRMS (ESI) $\mathrm{C}_{24} \mathrm{H}_{36} \mathrm{O}_{4}+\mathrm{Na}$ requires 411.2511 , found 411.2514 .

( \pm -tert-Butyl I(1R,2R,3R)-2-heptyl-3-[2-(2-methoxyphenyl)-2oxoethyl]cyclopropane-1-carboxylate $5 \mathrm{~b}$ and $( \pm)$-tert-butyl $[(1 R$, $\mathbf{2 S , 3 S ) - 2 - h e p t y l - 3 - ( 2 - m e t h o x y b e n z o y l ) c y c l o p r o p y l ] a c e t a t e \quad 9 b .}$ Cyclopropane 5b: $R_{\mathrm{f}}$ 0.49; (3.15 g, 50\%) as a colourless oil $v_{\max }($ film $) / \mathrm{cm}^{-1} 1716,1681,1599,1583,1486,1367,1246,1154$, 757; $\delta_{\mathrm{H}}\left(600 \mathrm{MHz} ; \mathrm{CDCl}_{3} ; \mathrm{Me}_{4} \mathrm{Si}\right) 0.87(3 \mathrm{H}, \mathrm{t}, J 7.2 \mathrm{~Hz}, \mathrm{Me})$, 1.04-1.09 (1H, m, C(3)H), 1.20-1.32 (10H, m, $\left.5 \times \mathrm{CH}_{2}\right), 1.34-1.50$ $\left(2 \mathrm{H}, \mathrm{m}, \mathrm{C}(2) \mathrm{H}\right.$ and $\left.\mathrm{CH}_{2}\right), 1.43\left(9 \mathrm{H}, \mathrm{s},{ }^{t} \mathrm{Bu}\right), 1.55-1.62(2 \mathrm{H}, \mathrm{m}$, $\mathrm{C}(1) \mathrm{H}$ and $\left.\mathrm{CH}_{2}\right), 2.76\left(1 \mathrm{H}\right.$, dd, $J 16.2$ and $\left.7.8 \mathrm{~Hz}, \mathrm{C}(1) \mathrm{HCH}_{2}\right)$, $3.12\left(1 \mathrm{H}, \mathrm{dd}, J 16.2\right.$ and $\left.6.0 \mathrm{~Hz}, \mathrm{C}(1) \mathrm{HCH}_{2}\right), 3.88(3 \mathrm{H}, \mathrm{s}, \mathrm{OMe})$, 6.94-6.95 (1H, m, Ph), 6.98-7.01 (1H, m, Ph), 7.43-7.45 (1H, $\mathrm{m}, \mathrm{Ph}), 7.65-7.66(1 \mathrm{H}, \mathrm{m}, \mathrm{Ph}) ; \delta_{\mathrm{C}}\left(75 \mathrm{MHz} ; \mathrm{CDCl}_{3} ; \mathrm{Me}_{4} \mathrm{Si}\right) 14.0$, 22.5, 22.6, 26.0, 26.7, 28.1, 29.0, 29.2, 29.3, 29.4, 31.8, 47.2, 55.3, $79.9,111.3,120.6,128.2,130.2,133.3,158.3,171.5,201.2 ; \mathrm{m} / \mathrm{z}$ (EI) $388\left(\mathrm{M}^{+}, 6 \%\right), 359$ (1), 331 (16), 287 (2), 273 (18), 247 (2), 219 (2), 175 (2), 150 (5), 135 (100), 92 (4), 57 (15), 42 (9); HRMS (ESI) $\mathrm{C}_{24} \mathrm{H}_{36} \mathrm{O}_{4}+\mathrm{Na}$ requires 411.2511 , found 411.2504. Cyclopropane 9b: $R_{\mathrm{f}} 0.41 ;(1.40 \mathrm{~g}, 22 \%)$ as a colourless oil $v_{\max }($ film $) / \mathrm{cm}^{-1} 1718$, $1708,1603,1585,1497,1459,1365,1250,1146 ; \delta_{\mathrm{H}}(600 \mathrm{MHz}$; $\left.\mathrm{CDCl}_{3} ; \mathrm{Me}_{4} \mathrm{Si}\right) 0.87(3 \mathrm{H}, \mathrm{t}, J 6.6 \mathrm{~Hz}, \mathrm{Me}), 1.11\left(9 \mathrm{H}, \mathrm{s},{ }^{t} \mathrm{Bu}\right)$, 1.22-1.32 (8H, m, $\left.4 \times \mathrm{CH}_{2}\right), 1.57-1.62\left(2 \mathrm{H}, \mathrm{m}, \mathrm{CH}_{2}\right), 1.82(1 \mathrm{H}$, dd, $J 9.6$ and $5.4 \mathrm{~Hz}, \mathrm{C}(3) \mathrm{H}), 2.19(1 \mathrm{H}$, dddd, $J$ 8.4, 7.2, 5.4 and $5.4 \mathrm{~Hz}, \mathrm{C}(1) \mathrm{H}), 2.24(1 \mathrm{H}$, dd, $J 9.6$ and $7.2 \mathrm{~Hz}, \mathrm{C}(2) \mathrm{H}), 2.36$ $\left(1 \mathrm{H}, \mathrm{dd}, J 16.8\right.$ and $\left.8.4 \mathrm{~Hz}, \mathrm{C}(1) \mathrm{HCH}_{2} \mathrm{C}(\mathrm{O})\right), 2.42-2.51(2 \mathrm{H}, \mathrm{m}$, $\left.\mathrm{CH}_{2} \mathrm{C}(\mathrm{O})\right), 2.76\left(1 \mathrm{H}, \mathrm{dd}, J 16.8\right.$ and $\left.5.4 \mathrm{~Hz}, \mathrm{C}(1) \mathrm{HCH}_{2} \mathrm{C}(\mathrm{O})\right)$, 6.77-6.79 (1H, m, Ph), 6.89-6.91 (1H, m, Ph), 7.18-7.26 (1H, m, $\mathrm{Ph}), 7.36-7.38(1 \mathrm{H}, \mathrm{m}, \mathrm{Ph}) ; \delta_{\mathrm{C}}\left(150 \mathrm{MHz} ; \mathrm{CDCl}_{3} ; \mathrm{Me}_{4} \mathrm{Si}\right)$ 14.0, 19.9, 22.5, 23.6, 27.6, 28.2, 29.0, 29.1, 31.6, 42.6, 46.0, 55.1, 79.7, 109.4, 120.0, 125.0, 127.8, 130.6, 158.5, 169.9, 209.6; $\mathrm{m} / \mathrm{z}$ (EI) 388 $\left(\mathrm{M}^{+}, 9 \%\right), 342$ (2), 315 (12), 287 (3), 273 (1), 247 (1), 205 (11), 190 (80), 160 (41), 127 (49), 91 (14), 57 (100), 42 (54); Anal. Calcd for $\mathrm{C}_{20} \mathrm{H}_{28} \mathrm{O}_{4}$ requires C, 74.19; $\mathrm{H}, 9.34$ : found $\mathrm{C}, 74.35 ; \mathrm{H}, 9.29 \%$.

( \pm -tert-Butyl I(1R,2R,3R)-2-heptyl-3-(2-phenyl-2-oxoethyl)cyclopropane-1-carboxylate $5 \mathrm{~d}$ and $( \pm)$-tert-butyl $[(1 R, 2 S, 3 S)$ 2-heptyl-3-benzoylcyclopropyl|acetate 9d. Cyclopropane 5d: $R_{\mathrm{f}}$ $0.43 ;(3.07 \mathrm{~g}, 52 \%)$ as a colourless oil $v_{\max }($ film $) / \mathrm{cm}^{-1} 1718,1690$, $1598,1581,1449,1366,1212,1153 ; \delta_{\mathrm{H}}\left(600 \mathrm{MHz} ; \mathrm{CDCl}_{3} ; \mathrm{Me}_{4} \mathrm{Si}\right)$ $0.87(3 \mathrm{H}, \mathrm{t}, J 7.2 \mathrm{~Hz}, \mathrm{Me}), 1.11(1 \mathrm{H}$, dddd, $J 9.0,7.8,6.6$ and $6.6 \mathrm{~Hz}, \mathrm{C}(3) \mathrm{H}), 1.20-1.33\left(10 \mathrm{H}, \mathrm{m}, 5 \times \mathrm{CH}_{2}\right), 1,45\left(9 \mathrm{H}, \mathrm{s},{ }^{t} \mathrm{Bu}\right)$, 
1.46-1.52 (2H, m, C(2)H and $\left.\mathrm{CH}_{2}\right), 1.59-1.68(2 \mathrm{H}, \mathrm{m}, \mathrm{C}(1) \mathrm{H}$ and $\left.\mathrm{CH}_{2}\right), 2.72\left(1 \mathrm{H}, \mathrm{dd}, J 16.2\right.$ and $\left.7.8 \mathrm{~Hz}, \mathrm{CH}_{2} \mathrm{C}(\mathrm{O}) \mathrm{Ph}\right), 3.18$ $\left(1 \mathrm{H}, \mathrm{dd}, J 16.2\right.$ and $\left.5.4 \mathrm{~Hz}, \mathrm{CH}_{2} \mathrm{C}(\mathrm{O}) \mathrm{Ph}\right), 7.44-7.47(2 \mathrm{H}, \mathrm{m}$, $\mathrm{Ph}), 7.54-7.57$ (1H, m, Ph), 7.91-7.93 (2H, m, Ph); $\delta_{\mathrm{C}}(150 \mathrm{MHz}$; $\left.\mathrm{CDCl}_{3} ; \mathrm{Me}_{4} \mathrm{Si}\right)$ 14.1, 22.3, 22.6, 26.3, 26.7, 28.2, 29.1, 29.26, $29.32,29.5,31.8,42.2,80.2,128.1,128.6,133.0,136.7,171.3$, 198.7; $\mathrm{m} / \mathrm{z}$ (EI) 359 [(M + H) $\left.{ }^{+}, 4 \%\right], 285$ (8), 120 (23), 105 (100), 57 (32); HRMS (ESI) $\mathrm{C}_{23} \mathrm{H}_{34} \mathrm{O}_{3}+\mathrm{Na}$ requires 381.2406, found 381.2400. Cyclopropane 9d: $R_{\mathrm{f}}$ 0.36; $(1.06 \mathrm{~g}, 18 \%)$ as a colourless oil $v_{\max }($ film $) / \mathrm{cm}^{-1} 1723,1714,1605,1367,1146 ; \delta_{\mathrm{H}}(600 \mathrm{MHz}$; $\left.\mathrm{CDCl}_{3} ; \mathrm{Me}_{4} \mathrm{Si}\right) 0.87(3 \mathrm{H}, \mathrm{t}, J 7.2 \mathrm{~Hz}, \mathrm{Me}), 1.13\left(9 \mathrm{H}, \mathrm{s},{ }^{t} \mathrm{Bu}\right)$, 1.23-1.31 (8H, m, $\left.4 \times \mathrm{CH}_{2}\right), 1.58-1.62\left(2 \mathrm{H}, \mathrm{m}, \mathrm{CH}_{2}\right), 1.81(1 \mathrm{H}$, $\mathrm{dd}, J 9.6$ and $4.8 \mathrm{~Hz}, \mathrm{C}(3) \mathrm{H}), 2.27(1 \mathrm{H}$, dddd, $J$ 8.4, 6.6, 5.4 and $4.8 \mathrm{~Hz}, \mathrm{C}(1) \mathrm{H}), 2.33-2.37\left(2 \mathrm{H}, \mathrm{m}, \mathrm{C}(2) \mathrm{H}\right.$ and $\left.\mathrm{C}(2) \mathrm{HCH}_{2} \mathrm{C}(\mathrm{O})\right)$, 2.45-2.48 (2H, m, $\left.\mathrm{CH}_{2} \mathrm{C}(\mathrm{O}) \mathrm{CH}_{2} \mathrm{C}(2) \mathrm{H}\right), 2.76(1 \mathrm{H}, \mathrm{dd}, J 16.8$ and $\left.5.4 \mathrm{~Hz}, \mathrm{C}(2) \mathrm{HCH}_{2} \mathrm{C}(\mathrm{O})\right), 7.17-7.20(1 \mathrm{H}, \mathrm{m}, \mathrm{Ph}), 7.24-7.27$ $(2 \mathrm{H}, \mathrm{m}, \mathrm{Ph}), 7.32-7.34(2 \mathrm{H}, \mathrm{m}, \mathrm{Ph}) ; \delta_{\mathrm{C}}\left(150 \mathrm{MHz} ; \mathrm{CDCl}_{3} ; \mathrm{Me}_{4} \mathrm{Si}\right)$ 14.0, 19.6, 22.6, 23.7, 27.7, 28.9, 29.0, 29.2, 31.6, 32.3, 42.6, 45.9, $80.3,126.6,127.9,129.5,136.3,169.5,209.4 ; \mathrm{m} / z(\mathrm{EI}) 358\left(\mathrm{M}^{+}<\right.$ 1\%), 302 (63), 284 (34), 257 (12), 57 (100), 41 (45); HRMS (ESI) $\mathrm{C}_{23} \mathrm{H}_{34} \mathrm{O}_{3}+\mathrm{Na}$ requires 381. 2406, found 381.2396.

(土)-tert-Butyl 2-[(1S,2R,3S)-2-heptyl-3-(2-methoxybenzoyl)cyclopropyl]acetate $10 \mathrm{~b}$ and $( \pm)$-tert-butyl $2-[(1 S, 2 R, 3 R)-2$-heptyl-3(2-methoxybenzoyl)cyclopropyl]acetate 11b

To a solution of 1,2-dioxine $\mathbf{6 b}$ (1 equiv.) in anhydrous dichloromethane $(53 \mathrm{ml}$ per $\mathrm{g}$ of $\mathbf{6 b})$ was added lithium bromide ( 0.1 equiv.) and (tert-butoxycarbonylmethylene)triphenylphosphorane (1.4 equiv.). The mixture was stirred at ambient temperature for 30 days. The solution was concentrated and the resulting residue purified by column chromatography using 3:17 ethyl acetate-hexanes as eluent to give cyclopropane $\mathbf{1 0 b}: R_{\mathrm{f}} 0.57$; (47 mg, 35\% based on $117 \mathrm{mg}$ recovered starting material) as a colourless oil $v_{\max }($ film $) / \mathrm{cm}^{-1} 1732,1726,1667,1660,1598,1485$, $1463,1435,1392,1367,1282,1246,1152 ; \delta_{\mathrm{H}}\left(300 \mathrm{MHz} ; \mathrm{CDCl}_{3}\right.$; $\left.\mathrm{Me}_{4} \mathrm{Si}\right) 0.86(3 \mathrm{H}, \mathrm{t}, J 7.2 \mathrm{~Hz}, \mathrm{Me}), 1.25-1.53(21 \mathrm{H}, \mathrm{m}), 1.64$ $1.68(2 \mathrm{H}, \mathrm{m}), 1.85(1 \mathrm{H}$, pentet, $J 7.8 \mathrm{~Hz}), 2.78-2.81(2 \mathrm{H}, \mathrm{m}$, $\left.\mathrm{CH}_{2} \mathrm{CO}_{2}{ }^{t} \mathrm{Bu}\right), 3.87$ (3H, s, OMe), 6.92-6.99 (2H, m, PH), 7.38$7.46(2 \mathrm{H}, \mathrm{m}, \mathrm{Ph}) ; \delta_{\mathrm{C}}\left(75 \mathrm{MHz} ; \mathrm{CDCl}_{3} ; \mathrm{Me}_{4} \mathrm{Si}\right)$ 14.1, 22.0, 22.6, 24.1, 28.1, 28.7, 29.1, 29.2, 29.3, 29.4, 29.7, 31.9, 55.5, 80.0, 111.4, $120.5,129.3,132.0,132.2,157.7,172.9,202.2 ; \mathrm{m} / \mathrm{z}$ (EI) $389[(\mathrm{M}+$ $\left.\mathrm{H})^{+}, 10 \%\right], 333$ (97), 273 (33), 135 (100), 57 (12); HRMS (ESI) $\mathrm{C}_{24} \mathrm{H}_{36} \mathrm{O}_{4}+\mathrm{H}$ requires 389.2692 , found 389.2690 and $\mathbf{1 1 b}: R_{\mathrm{f}} 0.46$; (65 mg, 58\% based on recovered starting material) as a colourless oil $v_{\max }(\mathrm{film}) / \mathrm{cm}^{-1} 1732,1727,1660,1651,1598,1485,1463,1438$, $1367,1283,1246,1154 ; \delta_{\mathrm{H}}\left(600 \mathrm{MHz} ; \mathrm{CDCl}_{3} ; \mathrm{Me}_{4} \mathrm{Si}\right) 0.87(3 \mathrm{H}, \mathrm{t}, J$ 7.2 Hz, Me), 1.21-1.34 (10H, m, $\left.5 \times \mathrm{CH}_{2}\right), 1.37-1.51\left(11 \mathrm{H}, \mathrm{m}, \mathrm{CH}_{2}\right.$ and $\left.{ }^{t} \mathrm{Bu}\right), 1.79\left(1 \mathrm{H}\right.$, dddd, $J$ 9.0, 7.2, 7.2 and $\left.4.2 \mathrm{~Hz}, \mathrm{CHC}_{7} \mathrm{H}_{15}\right)$, $2.04\left(1 \mathrm{H}\right.$, dddd, $J$ 9.0, 7.2, 7.2 and $\left.4.2 \mathrm{~Hz}, \mathrm{CHCH}_{2} \mathrm{CO}_{2}{ }^{t} \mathrm{Bu}\right), 2.30$ $(1 \mathrm{H}, \mathrm{t}, J 4.2 \mathrm{~Hz}, \mathrm{CHC}(\mathrm{O})), 2.37(1 \mathrm{H}, \mathrm{dd}, J 15.0$ and $7.2 \mathrm{~Hz}$, $\left.\mathrm{CH}_{2} \mathrm{CO}_{2}{ }^{t} \mathrm{Bu}\right), 2.38\left(1 \mathrm{H}\right.$, dd, $J 15.0$ and $\left.7.2 \mathrm{~Hz}, \mathrm{CH}_{2} \mathrm{CO}_{2}{ }^{t} \mathrm{Bu}\right), 6.95-$ 7.00 (2H, m, Ph), 7.41-7.44 (1H, m, Ph), 7.55-7.57 (1H, m, Ph); $\delta_{\mathrm{C}}\left(150 \mathrm{MHz} ; \mathrm{CDCl}_{3} ; \mathrm{Me}_{4} \mathrm{Si}\right)$ 14.0, 22.6, 27.3, 27.9, 28.0, 29.2, 29.4, 31.2, 31.8, 34.6, 36.2, 55.5, 80.4, 111.5, 120.5, 129.7, 129.8, 132.8, 158.2, 171.6, 201.9; $\mathrm{m} / \mathrm{z}$ (EI) $389\left[(\mathrm{M}+\mathrm{H})^{+}, 13 \%\right], 333$ (41), 273 (39), 135 (100), 57 (29); HRMS (ESI) $\mathrm{C}_{24} \mathrm{H}_{36} \mathrm{O}_{4}+\mathrm{H}$ requires 389.2692 , found 389.2691 .

\section{Optimized procedure for the synthesis of $5 \mathrm{a}$ and $5 \mathrm{~b}$}

To a solution of 1,2-dioxine $\mathbf{6 a}$ or $\mathbf{6 b}$ (1 equiv.) in anhydrous dichloromethane (53 $\mathrm{ml}$ per $\mathrm{g}$ of $\mathbf{6})$ was added lithium bromide (1.1 equiv.), (tert-butoxycarbonylmethylene)triphenylphosphorane (1.4 equiv.) followed by triethylamine ( 2 drops for every $50 \mathrm{mg}$ of 6). The mixture was stirred at ambient temperature for 2 days (Table 1). The solution was concentrated and the resulting residue purified by column chromatography using 3:17 ethyl acetate-hexanes as eluent to give cyclopropanes $\mathbf{5 a}$ or $\mathbf{5 b}$.

\section{( \pm )-(1S,2R,3R)-2-Heptyl-3-[2-(4-methoxyphenyl)-2- oxoethyl]cyclopropane-1-carboxylic acid 16}

99\% Formic acid ( $2 \mathrm{ml}$ ) was added to cyclopropyl ester $\mathbf{5 a}$ (400 mg, $1.03 \mathrm{mmol}$ ) and the solution stirred for 3 hours. The formic acid was then removed in vacuo, the resulting crude acid $\mathbf{1 6}$ was dissolved in ethyl acetate and washed with $6 \% \mathrm{NaHCO}_{3}$ solution. The organics were then dried $\left(\mathrm{MgSO}_{4}\right)$, filtered and the volatiles removed in vacuo to yield cyclopropyl acid 16 (304 mg, 89\%) as a colourless crystalline solid. Mp $116-117^{\circ} \mathrm{C}$ (diethyl ether); $v_{\max }($ film $) / \mathrm{cm}^{-1} 2601,1688,1682,1668,1602,1574,1171,1027$, 925; $\delta_{\mathrm{H}}\left(600 \mathrm{MHz} ; \mathrm{CDCl}_{3} ; \mathrm{Me}_{4} \mathrm{Si}\right) 0.87(3 \mathrm{H}, \mathrm{t}, J 6.6 \mathrm{~Hz}, \mathrm{Me})$, 1.21-1.29 (12H, m), 1.54-1.67 (3H, m), 1.75-1.79 (1H, m), 2.79 $\left(1 \mathrm{H}, \mathrm{dd}, J 16.2\right.$ and $\left.7.2 \mathrm{~Hz}, \mathrm{CH}_{2}\right), 3.09(1 \mathrm{H}, \mathrm{dd}, J 16.2$ and $6.6 \mathrm{~Hz}$, $\mathrm{CH}_{2}$ ), 3.87 (3H, s, OMe), 6.93-6.94 (2H, m, Ph), 7.90-7.91 (2H, $\mathrm{m}, \mathrm{Ph}) ; \delta_{\mathrm{C}}\left(75 \mathrm{MHz} ; \mathrm{CDCl}_{3} ; \mathrm{Me}_{4} \mathrm{Si}\right) 14.1,22.6,24.1,24.9,26.6$, 29.17, 29.22, 29.3, 30.4, 31.8, 41.8, 55.5, 113.8, 129.7, 130.4, 163.6, 178.3, 196.8; $m / z$ (EI) $332\left(\mathrm{M}^{+}, 16 \%\right), 315$ (65), 273 (45), 247 (4), 219 (21), 187 (11), 150 (31), 135 (100), 121 (12), 92 (19), 77 (24), 55 (17), 41 (41); HRMS (ESI) $\mathrm{C}_{20} \mathrm{H}_{28} \mathrm{O}_{4}+\mathrm{Na}$ requires 355.1885, found 355.1879 .

\section{( \pm )-2-[(1 $R, 2 S)$-2-Heptylcyclopropyl]-1-(4-methoxyphenyl)-1- ethanone 4}

To a solution of cyclopropane acid $16(384 \mathrm{mg}, 1.15 \mathrm{mmol})$ in anhydrous dichloromethane $(30 \mathrm{ml})$ at $0{ }^{\circ} \mathrm{C}$ under a nitrogen atmosphere and protected from light was added 2-mercaptopyridine $N$ oxide (147 mg, $1.15 \mathrm{mmol}$ ) followed by DCC (238 mg, $1.15 \mathrm{mmol}$ ). The reaction mixture was then stirred for 3 hours under the stated conditions. After this time the precipitate was filtered off and washed with cold dichloromethane. The volatiles were removed in vacuo and the crude oil reconstituted in anhydrous benzene $(45 \mathrm{ml})$. The solution was then degassed and tert-butyl thiol $(0.26 \mathrm{ml}$, $2.31 \mathrm{mmol}$ ) added; irradiation of the solution was preformed with 1 sun lamp (at a distance of $30 \mathrm{~cm}$ from the reaction vessel) under a nitrogen atmosphere for 2 hours. The volatiles were then removed in vacuo and the crude oil purified by column chromatography using 3:1 dichloromethane-hexanes as eluent to give cyclopropane 4. $R_{\mathrm{f}} 0.50 ;(287 \mathrm{mg}, 86 \%)$ as a colourless oil. $v_{\max }($ film $) / \mathrm{cm}^{-1} 1681$, $1602,1577,1510,1259,1170,1033 ; \delta_{\mathrm{H}}\left(300 \mathrm{MHz} ; \mathrm{CDCl}_{3} ; \mathrm{Me}_{4} \mathrm{Si}\right)$ $0.31-0.36\left(2 \mathrm{H}, \mathrm{m}, \mathrm{CH}_{2}\right), 0.51-0.62(1 \mathrm{H}, \mathrm{m}, \mathrm{CH}), 0.82-0.90(4 \mathrm{H}, \mathrm{m}$, $\mathrm{Me}$ and $\mathrm{CH}), 1.19-1.39\left(12 \mathrm{H}, \mathrm{m}, 6 \times \mathrm{CH}_{2}\right), 2.82(2 \mathrm{H}, \mathrm{d}, J 6.9 \mathrm{~Hz}$, $\left.\mathrm{CH}_{2} \mathrm{C}(\mathrm{O})\right), 3.86$ (3H, s, OMe), 6.91-6.94 (2H, m, Ph), 7.91-7.94 $(2 \mathrm{H}, \mathrm{m}, \mathrm{Ph}) ; \delta_{\mathrm{C}}\left(75 \mathrm{MHz} ; \mathrm{CDCl}_{3} ; \mathrm{Me}_{4} \mathrm{Si}\right) 11.9,14.1,14.5,18.9$, 22.6, 29.3, 29.36, 29.39, 31.8, 34.0, 43.1, 55.4, 113.7, 130.2, 130.4, 163.3, 198.6; $\mathrm{m} / \mathrm{z}$ (EI) $288\left(\mathrm{M}^{+}, 51 \%\right), 273$ (79), 175 (12), 135 (65), 92 (12), 77 (11), 64 (11), 55 (13), 41 (100); HRMS (ESI) $\mathrm{C}_{19} \mathrm{H}_{28} \mathrm{O}_{2}+$ $\mathrm{Na}$ requires 311.1987 , found 311.1984 . 


\section{(土)-4-Methoxyphenyl 2-[(1R,2S)-2-heptylcyclopropyl]acetate 3}

To a solution of cyclopropane 4 (986 mg, $3.42 \mathrm{mmol})$ in dichloromethane $(10 \mathrm{ml})$ was added $70 \%$ MCPBA $(1.685 \mathrm{~g}$, $6.83 \mathrm{mmol}$ ) and the mixture was stirred for 2 days. Dichloromethane $(20 \mathrm{ml})$ was then added and the solution extracted with saturated $\mathrm{Na}_{2} \mathrm{~S}_{2} \mathrm{O}_{3}$ followed by saturated $\mathrm{NaHCO}_{3}$. The organic layers were combined, dried $\left(\mathrm{MgSO}_{4}\right)$, filtered and the volatiles removed in vacuo to yield $947 \mathrm{mg}, 91 \%$ of cyclopropyl ester 3 as a colourless oil. $v_{\max }$ (film)/cm $\mathrm{cm}^{-1} 1760,1608,1598,1506,1249,1196$, $1036 ; \delta_{\mathrm{H}}\left(300 \mathrm{MHz} ; \mathrm{CDCl}_{3} ; \mathrm{Me}_{4} \mathrm{Si}\right) 0.39-0.47\left(2 \mathrm{H}, \mathrm{m}, \mathrm{CH}_{2}\right), 0.63-$ $0.74(1 \mathrm{H}, \mathrm{m}, \mathrm{CH}), 0.88-0.98$ (4H, m, Me and $\mathrm{CH}), 1.23-1.50(12 \mathrm{H}$, $\left.\mathrm{m}, 6 \times \mathrm{CH}_{2}\right), 2.43\left(1 \mathrm{H}, \mathrm{dd}, J 15.3\right.$ and $\left.7.5 \mathrm{~Hz}, \mathrm{CH}_{2} \mathrm{C}(\mathrm{O})\right), 2.53$ $\left(1 \mathrm{H}, \mathrm{dd}, J 15.3\right.$ and $\left.6.6 \mathrm{~Hz}, \mathrm{CH}_{2} \mathrm{C}(\mathrm{O})\right), 3.84(3 \mathrm{H}, \mathrm{s}, \mathrm{OMe}), 6.90$ $7.00(2 \mathrm{H}, \mathrm{m}, \mathrm{Ph}), 7.03-7.08(2 \mathrm{H}, \mathrm{m}, \mathrm{Ph}) ; \delta_{\mathrm{C}}\left(75 \mathrm{MHz} ; \mathrm{CDCl}_{3}\right.$; $\mathrm{Me}_{4} \mathrm{Si}$ ) 11.7, 14.1, 14.3, 18.8, 22.7, 29.3, 29.4, (masked carbon), 31.9, 33.9, 39.1, 55.6, 114.4, 122.3, 144.4, 157.2, 172.0; $\mathrm{m} / z(\mathrm{EI})$ $304\left(\mathrm{M}^{+}, 51 \%\right), 273$ (7), 203 (7), 175 (31), 163 (5), 135 (17), 124 (100), 109 (9), 82 (10), 55 (14), 41 (15); HRMS (ESI) $\mathrm{C}_{19} \mathrm{H}_{28} \mathrm{O}_{3}+$ $\mathrm{Na}$ requires 327.1936 , found 327.1936 .

\section{(土)-Methyl 2-[(1S,2R)-2-heptylcyclopropyl]acetate 17}

To a solution of cyclopropyl ester $3(1.031 \mathrm{~g}, 3.39 \mathrm{mmol})$ in anhydrous methanol $(20 \mathrm{ml})$ was added concentrated $\mathrm{H}_{2} \mathrm{SO}_{4}$ ( 1 drop) and the solution refluxed for 16 hours under a nitrogen atmosphere. $\mathrm{NaHCO}_{3}(50 \mathrm{mg}, 0.6 \mathrm{mmol})$ was then added and the volatiles removed in vacuo until approximately $5 \mathrm{ml}$ remained. Dichloromethane $(20 \mathrm{ml})$ was then added and the solution extracted with saturated $\mathrm{NaHCO}_{3}$, dried $\left(\mathrm{MgSO}_{4}\right)$, filtered and the volatiles removed in vacuo. Column chromatography of the crude residue using 7:3 dichloromethane-hexanes as eluent gave cyclopropyl ester $17, R_{\mathrm{f}} 0.63$; (670 $\left.\mathrm{mg}, 93 \%\right)$, as a colourless oil. $v_{\max }($ film $) / \mathrm{cm}^{-1} 1746,1436,1256,1171,1020 ; \delta_{\mathrm{H}}\left(600 \mathrm{MHz} ; \mathrm{CDCl}_{3}\right.$; $\left.\mathrm{Me}_{4} \mathrm{Si}\right)$ 0.28-0.32 (2H, m, $\left.\mathrm{CH}_{2}\right), 0.50-0.55(1 \mathrm{H}, \mathrm{m}, \mathrm{CH}), 0.74$ $0.79(1 \mathrm{H}, \mathrm{m}, \mathrm{CH}), 0.88(3 \mathrm{H}, \mathrm{t}, J 6.6 \mathrm{~Hz}, \mathrm{Me}), 1.16-1.23(1 \mathrm{H}$, m), 1.23-1.31 (9H, m), 1.34-1.39 (2H, m, $\left.\mathrm{CH}_{2}\right), 2.21(1 \mathrm{H}, \mathrm{dd}, J$ 15.6 and $\left.7.2 \mathrm{~Hz}, \mathrm{CH}_{2} \mathrm{CO}_{2} \mathrm{Me}\right), 2.23(1 \mathrm{H}$, dd, $J 15.6$ and $7.2 \mathrm{~Hz}$, $\left.\mathrm{CH}_{2} \mathrm{CO}_{2} \mathrm{Me}\right), 3.68(3 \mathrm{H}, \mathrm{s}, \mathrm{OMe}) ; \delta_{\mathrm{C}}\left(75 \mathrm{MHz} ; \mathrm{CDCl}_{3} ; \mathrm{Me}_{4} \mathrm{Si}\right) 11.6$, 14.1, 14.2, 18.6, 22.7, 29.3, 29.4, (masked carbon), 31.9, 33.9, 38.9, 51.5, 173.8; $m / z(\mathrm{EI}) 213\left([\mathrm{M}+\mathrm{H}]^{+}, 13 \%\right), 197$ (7), $183(8), 180$ (66), 169 (14), 152 (3), 138 (50), 128 (13), 110 (16), 101 (61), 83 (57), 74 (94), 59 (85), 55 (87), 41 (100); HRMS (ESI) $\mathrm{C}_{19} \mathrm{H}_{24} \mathrm{O}_{3}$ requires 212.1776 , found 212.1771 .

\section{(土)-2-[(1R,2S)-2-Heptylcyclopropyl]acetic acid 2}

To a solution of cyclopropyl ester $17(1.209 \mathrm{~g}, 5.69 \mathrm{mmol})$ in water and methanol (15 ml, 1:4 v/v) was added aqueous $\mathrm{KOH}(4 \mathrm{ml}, 2 \mathrm{M})$ and the solution stirred for 6 hours. After this time, water $(10 \mathrm{ml})$ was added and the solution was acidified (conc. $\mathrm{HCl}$ ) to $\mathrm{pH} 1$. Following acidification the solution was extracted with ether, the organics were dried $\left(\mathrm{MgSO}_{4}\right)$, filtered and the volatiles removed in vacuo to give cyclopropyl acid $2(1.05,93 \%)$ as a colourless oil. $v_{\max }($ film $) / \mathrm{cm}^{-1} 2673,1714,1416,1304,1223,940 ; \delta_{\mathrm{H}}(600 \mathrm{MHz}$; $\left.\mathrm{CDCl}_{3} ; \mathrm{Me}_{4} \mathrm{Si}\right)$ 0.31-0.35 (2H, m, CH$), 0.53-0.58(1 \mathrm{H}, \mathrm{m}, \mathrm{CH})$, $0.75-0.80(1 \mathrm{H}, \mathrm{m}, \mathrm{CH}), 0.88(3 \mathrm{H}, \mathrm{t}, J 7.2 \mathrm{~Hz}, \mathrm{Me}), 1.21-1.31(10 \mathrm{H}$, $\left.\mathrm{m}, 5 \times \mathrm{CH}_{2}\right), 1.35-1.40\left(2 \mathrm{H}, \mathrm{m}, \mathrm{CH}_{2}\right), 2.25(1 \mathrm{H}, \mathrm{dd}, J 16.2$ and 6.7 Hz, CH $\left.\mathrm{CO}_{2} \mathrm{H}\right), 2.27\left(1 \mathrm{H}, \mathrm{dd}, J 16.2\right.$ and $\left.7.7 \mathrm{~Hz}, \mathrm{CH}_{2} \mathrm{CO}_{2} \mathrm{H}\right)$; $\delta_{\mathrm{C}}\left(75 \mathrm{MHz} ; \mathrm{CDCl}_{3} ; \mathrm{Me}_{4} \mathrm{Si}\right) 11.6,14.0,14.1,18.7,22.7,29.32$,
29.35, 29.4, 31.9, 33.8, 38.8, 179.2; $\mathrm{m} / \mathrm{z}(\mathrm{EI}) 199\left([\mathrm{M}+\mathrm{H}]^{+}, 100 \%\right)$, 181 (64), 163 (20), 151 (6), 138 (63), 123 (17), 112 (34), 97 (63), 83 (98), 82 (53), 69 (45), 55 (36), 41 (53); HRMS (ESI) $\mathrm{C}_{12} \mathrm{H}_{22} \mathrm{O}_{2}-\mathrm{H}$ requires 197.1542 , found 197.1540 .

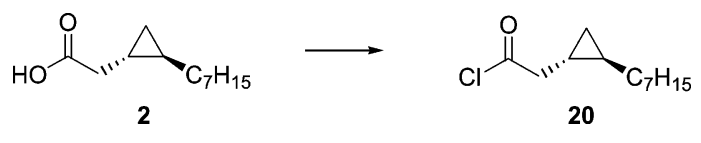

\section{( \pm )-2-[(1S,2R)-2-Heptylcyclopropyl]ethanoyl chloride 20}

To a solution of cyclopropyl acid 2 (413 mg, $2.08 \mathrm{mmol})$ and DMF (1 drop) in anhydrous dichloromethane $(15 \mathrm{ml})$ at $0{ }^{\circ} \mathrm{C}$ under a nitrogen atmosphere was added oxalyl chloride $(0.55 \mathrm{ml}$, $6.25 \mathrm{mmol})$ as a solution in anhydrous dichloromethane $(15 \mathrm{ml})$. The solution was then stirred for 2 hours at $0{ }^{\circ} \mathrm{C}$ then allowed to attain ambient temperature and stirred for a further 2 hours. The volatiles were then removed in vacuo to give crude acid chloride $\mathbf{2 0}$, which was used without further purification. $\delta_{\mathrm{H}}\left(300 \mathrm{MHz} ; \mathrm{CDCl}_{3}\right.$; $\left.\mathrm{Me}_{4} \mathrm{Si}\right)$ 0.37-0.45 (2H, m, $\left.\mathrm{CH}_{2}\right), 0.59-0.65$ (1H, m, CH), 0.82-0.91 (4H, m, Me and $\mathrm{CH}), 1.21-1.38\left(12 \mathrm{H}, \mathrm{m}, 6 \times \mathrm{CH}_{2}\right), 2.75(1 \mathrm{H}, \mathrm{dd}$, $J 16.8$ and $\left.7.1 \mathrm{~Hz}, \mathrm{CH}_{2} \mathrm{C}(\mathrm{O}) \mathrm{Cl}\right), 2.80(1 \mathrm{H}, \mathrm{dd}, J 16.8$ and $6.8 \mathrm{~Hz}$, $\left.\mathrm{CH}_{2} \mathrm{C}(\mathrm{O}) \mathrm{Cl}\right)$.

\section{(4S)-4-Benzyl-3-2-[(1S,2R)-2-heptylcyclopropyl]acetyl-1,3- oxazolan-2-one 18 and $(4 S)$-4-benzyl-3-2-[(1R,2S)-2-heptyl- cyclopropyl|acetyl-1,3-oxazolan-2-one 19}

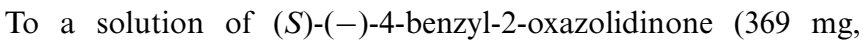
$2.08 \mathrm{mmol})$ in anhydrous THF $(20 \mathrm{ml})$ at $-78^{\circ} \mathrm{C}$ under a nitrogen atmosphere was added $n \mathrm{BuLi}(1.1 \mathrm{ml}, 1.88 \mathrm{M})$ and the solution stirred for 0.5 hours. After this time the solution of acid chloride 20 (synthesized from $413 \mathrm{mg}, 2.08 \mathrm{mmol}$ of acid 2) in anhydrous THF $(15 \mathrm{ml})$ was added dropwise and the solution stirred at $-78^{\circ} \mathrm{C}$ for 4 hours. The reaction was then quenched with saturated $\mathrm{NH}_{4} \mathrm{Cl}$, diluted with water and extracted with dichloromethane. The organics were dried $\left(\mathrm{MgSO}_{4}\right)$, filtered and concentrated in vacuo to give crude oxazolidinones. Oxazolidinones $\mathbf{1 8}$ and $\mathbf{1 9}$ were purified by column chromatography to give a combined yield of $789 \mathrm{mg}, 59 \%$. The oxazolidinones were resolved by successive column chromatography using 1:4 ethyl acetate-hexanes as eluent. Oxazolidinone 18 ( $1^{\text {st }}$ eluted): $R_{\mathrm{f}} 0.44 ; \mathrm{mp} 45-46{ }^{\circ} \mathrm{C}$; $v_{\max }($ nujol $) / \mathrm{cm}^{-1} 1790,1691,1225,1151,758 ; \delta_{\mathrm{H}}\left(300 \mathrm{MHz} ; \mathrm{CDCl}_{3}\right.$; $\left.\mathrm{Me}_{4} \mathrm{Si}\right)$ 0.33-0.41 (2H, m, CH $), 0.54-0.65$ (1H, m, CH), 0.83-0.94 $(4 \mathrm{H}, \mathrm{m}, \mathrm{Me}$ and $\mathrm{CH}), 1.18-1.44\left(12 \mathrm{H}, \mathrm{m}, 6 \times \mathrm{CH}_{2}\right), 2.78(1 \mathrm{H}, \mathrm{dd}$, $J 13.2$ and $\left.9.6 \mathrm{~Hz}, \mathrm{CH}_{2} \mathrm{Ph}\right), 2.81(1 \mathrm{H}, \mathrm{dd}, J 17.1$ and $7.2 \mathrm{~Hz}$, $\left.\mathrm{CH}_{2} \mathrm{C}(\mathrm{O})\right), 2.92\left(1 \mathrm{H}, \mathrm{dd}, J 17.1\right.$ and $\left.6.9 \mathrm{~Hz}, \mathrm{CH}_{2} \mathrm{C}(\mathrm{O})\right), 3.33(1 \mathrm{H}$, dd, $J 13.2$ and $\left.3.0 \mathrm{~Hz}, \mathrm{CH}_{2} \mathrm{Ph}\right), 4.16(1 \mathrm{H}$, dd, $J 9.0$ and $3.6 \mathrm{~Hz}$, $\left.\mathrm{CH}_{2} \mathrm{O}\right), 4.20\left(1 \mathrm{H}\right.$, dd, $J 9.0$ and $\left.7.2 \mathrm{~Hz}, \mathrm{CH}_{2} \mathrm{O}\right), 4.69(1 \mathrm{H}$, dddd, $J$ 9.6, 7.2, 3.6 and $3.0 \mathrm{~Hz}, \mathrm{NCH}), 7.20-7.37(5 \mathrm{H}, \mathrm{m}, \mathrm{Ph}) ; \delta_{\mathrm{C}}(75 \mathrm{MHz}$; $\mathrm{CDCl}_{3} ; \mathrm{Me}_{4} \mathrm{Si}$ ) 11.6, 13.7, 14.1, 18.5, 22.7, 29.3, 29.4, (masked carbon), 29.5, 31.9, 34.0, 38.0, 40.3, 55.2, 66.2, 127.3, 128.9, 129.4, 135.3, 153.5, 173.0; $\mathrm{m} / z$ (EI) $357\left(\mathrm{M}^{+}, 37 \%\right), 342$ (5), 272 (7), 244 (19), 219 (9), 178 (43), 152 (6), 134 (20), 117 (48), 91 (83), 55 (69), 41 (100); Anal. Calcd for $\mathrm{C}_{22} \mathrm{H}_{31} \mathrm{NO}_{3}$ requires $\mathrm{C}$, 73.92; $\mathrm{H}, 8.74$; $\mathrm{N}, 3.92$ : found $\mathrm{C}, 74.04 ; \mathrm{H}, 8.67 ; \mathrm{N}, 3.99 \%$. Oxazolidinone $19\left(2^{\text {nd }}\right.$ eluted): $R_{\mathrm{f}} 0.38 ; \mathrm{mp} 46-48{ }^{\circ} \mathrm{C} ; v_{\max }$ (nujol) $/ \mathrm{cm}^{-1} 1786,1696,1222$, 1118,$998 ; \delta_{\mathrm{H}}\left(300 \mathrm{MHz} ; \mathrm{CDCl}_{3} ; \mathrm{Me}_{4} \mathrm{Si}\right) 0.33-0.39\left(2 \mathrm{H}, \mathrm{m}, \mathrm{CH}_{2}\right)$, 0.56-0.66 (1H, m, CH), 0.83-0.94 (4H, m, Me and $\mathrm{CH}), 1.22-1.44$ $\left(12 \mathrm{H}, \mathrm{m}, 6 \times \mathrm{CH}_{2}\right), 2.76\left(1 \mathrm{H}, \mathrm{dd}, J 17.1\right.$ and $\left.7.2 \mathrm{~Hz}, \mathrm{CH}_{2} \mathrm{C}(\mathrm{O})\right)$, 
$2.78\left(1 \mathrm{H}, \mathrm{dd}, J 13.5\right.$ and $\left.9.6 \mathrm{~Hz}, \mathrm{CH}_{2} \mathrm{Ph}\right), 2.98(1 \mathrm{H}, \mathrm{dd}, J 17.1$ and $\left.6.6 \mathrm{~Hz}, \mathrm{CH}_{2} \mathrm{C}(\mathrm{O})\right), 3.32\left(1 \mathrm{H}, \mathrm{dd}, J 13.5\right.$ and $\left.3.3 \mathrm{~Hz}, \mathrm{CH}_{2} \mathrm{Ph}\right), 4.16$ $\left(1 \mathrm{H}, \mathrm{dd}, J 9.0\right.$ and $\left.3.6 \mathrm{~Hz}, \mathrm{CH}_{2} \mathrm{O}\right), 4.20(1 \mathrm{H}, \mathrm{dd}, J 9.0$ and $7.2 \mathrm{~Hz}$, $\left.\mathrm{CH}_{2} \mathrm{O}\right), 4.69$ (1H, dddd, $J=9.6,7.2,3.6$ and $\left.3.3 \mathrm{~Hz}, \mathrm{NCH}\right), 7.20$ $7.37(5 \mathrm{H}, \mathrm{m}, \mathrm{Ph}) ; \delta_{\mathrm{C}}\left(75 \mathrm{MHz} ; \mathrm{CDCl}_{3} ; \mathrm{Me}_{4} \mathrm{Si}\right) 11.5,13.7,14.1,18.7$, 22.7, 29.3, 29.4, (masked carbon), 31.9, 33.9, 38.0, 40.2, 55.2, 66.2, 127.3, 128.9, 129.4, 135.3, 153.5, 173.0; $\mathrm{m} / \mathrm{z}$ (EI) 357 (M+, 23\%), 342 (3), 272 (8), 244 (19), 219 (11), 181 (40), 134 (20), 117 (39), 91 (80), 55 (58), 41 (100); HRMS (ESI) $\mathrm{C}_{22} \mathrm{H}_{31} \mathrm{NO}_{3}+\mathrm{Na}$ requires 380.2202 , found 380.2199 .

\section{(-)-2-[(1S,2R)-2-Heptylcyclopropyl]acetic acid (-)-2}

To a solution of oxazolidinone $18(516 \mathrm{mg}, 1.44 \mathrm{mmol})$ in THF and water $(10 \mathrm{ml}, 3: 1 \mathrm{v} / \mathrm{v})$ at $0{ }^{\circ} \mathrm{C}$ was added $30 \%$ hydrogen peroxide $(1.31 \mathrm{ml}, 11.6 \mathrm{mmol})$ and $\mathrm{LiOH}(138 \mathrm{mg}, 5.76 \mathrm{mmol})$, and the solution was then stirred for 0.5 hours. After this time the reaction was quenched with $1.5 \mathrm{M} \mathrm{Na}_{2} \mathrm{SO}_{3}$ and extracted with dichloromethane, the addition of brine was necessary to break up the emulsions formed. The aqueous layer was then acidified (conc. $\mathrm{HCl}$ ) to $\mathrm{pH} 1$ and extracted with dichloromethane. The organics were dried $\left(\mathrm{MgSO}_{4}\right)$, filtered and concentrated in vacuo to give cyclopropyl acid (-)-2 (254 $\mathrm{mg}, 89 \%)$ as a colourless oil. $[\alpha]_{\mathrm{D}}^{20.5}$ $-14.0^{\circ}\left(\mathrm{CHCl}_{3}, c=0.01\right)$.

\section{(+)-2-[(1R,2S)-2-Heptylcyclopropyl]acetic acid (+)-2}

Procedure as for acid (-)-2 synthesis from oxazolidinone 19 $\left(275 \mathrm{mg}, 86^{\circ}\right)$ as a colourless oil. $[\alpha]_{\mathrm{D}}^{20.5}+14.0^{\circ}\left(\mathrm{CHCl}_{3}, c=0.01\right)$.

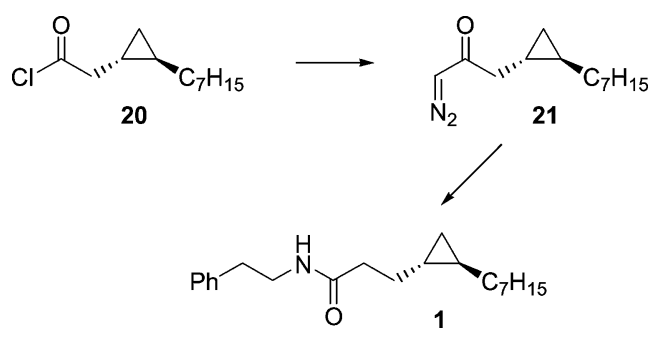

\section{( \pm -Grenadamide 1}

A solution of acid chloride 20 (synthesized from $250 \mathrm{mg}$, $1.26 \mathrm{mmol}$ of acid 2) in anhydrous ether $(8 \mathrm{ml})$ was added to a stirred solution of diazomethane ${ }^{12}(\sim 80 \mathrm{mg}, 1.9 \mathrm{mmol})$ in ether ( $\sim 60 \mathrm{ml})$ and triethylamine $(0.2 \mathrm{ml}, 1.43 \mathrm{mmol})$ at $0{ }^{\circ} \mathrm{C}$. The mixture was stirred for 0.5 hours, after which time half the volume of ether was evaporated under a stream of nitrogen. The precipitated $\mathrm{NEt}_{3} \cdot \mathrm{HCl}$ was then filtered off and washed with cold ether. The volatiles were removed in vacuo to give crude diazoketone 21. $R_{\mathrm{f}} 0.67$ (1:9 ether-dichloromethane). Diazoketone 21 was reconstituted in anhydrous, degassed THF (4 ml) and cooled to $-15{ }^{\circ} \mathrm{C}$, to this solution was added phenethylamine $(0.63 \mathrm{ml}, 5.04 \mathrm{mmol})$. The reaction was then protected from light and a solution of silver benzoate $(317 \mathrm{mg}, 1.39 \mathrm{mmol}$.) in triethylamine $(5.4 \mathrm{ml})$ was added. On complete addition of the silver benzoate solution the mixture was allowed to attain ambient temperature and was stirred for 16 hours. The volatiles were removed in vacuo and the residue purified by column chromatography using 1:1 ethyl acetate-hexanes as eluent to give racemic grenadamide, $R_{\mathrm{f}} 0.66$; (329 $\left.\mathrm{mg}, 83 \%\right)$, as a colourless solid, $\mathrm{mp} 54-56{ }^{\circ} \mathrm{C} ; v_{\max }$ (nujol) $/ \mathrm{cm}^{-1} 3312,1636,1549,1194,1024,747$; $\delta_{\mathrm{H}}\left(300 \mathrm{MHz} ; \mathrm{CDCl}_{3} ; \mathrm{Me}_{4} \mathrm{Si}\right) 0.12-0.21\left(2 \mathrm{H}, \mathrm{m}, \mathrm{CH}_{2}\right), 0.32-0.47$ $(2 \mathrm{H}, 2 \times \mathrm{CH}), 0.88(3 \mathrm{H}, \mathrm{t}, J 6.9 \mathrm{~Hz}, \mathrm{Me}), 1.05-1.38(12 \mathrm{H}, \mathrm{m}, 6 \times$ $\left.\mathrm{CH}_{2}\right), 1.44-1.57\left(2 \mathrm{H}, \mathrm{m}, \mathrm{CH}_{2}\right), 2.21\left(2 \mathrm{H}, \mathrm{t}, J 7.5 \mathrm{~Hz}, \mathrm{CH}_{2} \mathrm{C}(\mathrm{O})\right)$, $2.82\left(2 \mathrm{H}, \mathrm{t}, J 6.9 \mathrm{~Hz}, \mathrm{CH}_{2} \mathrm{Ph}\right), 3.52\left(2 \mathrm{H}, \mathrm{q}, J 6.9 \mathrm{~Hz}, \mathrm{NCH}_{2}\right), 5.78$ $\left(1 \mathrm{H}\right.$, br s, NH), $7.18-7.33(5 \mathrm{H}, \mathrm{m}, \mathrm{Ph}) ; \delta_{\mathrm{C}}\left(75 \mathrm{MHz} ; \mathrm{CDCl}_{3} ; \mathrm{Me}_{4} \mathrm{Si}\right)$ $11.7,14.1,18.1,18.8,22.6,29.3,29.4,29.6,30.4,31.8,34.1,35.6$, $36.7,40.5,126.4,128.6,128.7,138.8,173.2 ; \mathrm{m} / \mathrm{z}(\mathrm{EI}) 315.3\left(\mathrm{M}^{+}\right.$, 16\%), 286.3 (8), 267.4 (6), 244.2 (8), 230.1 (18), 217.3 (8), 182.2 (20), 163.1 (33), 132.1 (12), 115.1 (20), 140.1 (100), 72.1 (71), 55.1 (87), 43.1 (73); Anal. Calcd for $\mathrm{C}_{21} \mathrm{H}_{33} \mathrm{NO}$ requires $\mathrm{C}, 79.95 ; \mathrm{H}$, $10.54 ; \mathrm{N}, 4.44$ : found $\mathrm{C}, 79.98 ; \mathrm{H}, 10.68 ; \mathrm{N}, 4.50 \%$.

\section{(-)-( $R, R)$-Grenadamide (-)-1}

Procedure as for $( \pm)$-grenadamide synthesis from (-)-2 (296 mg, $80 \%)$ as a colourless solid. $[\alpha]_{\mathrm{D}}^{20.5}-12.0^{\circ}\left(\mathrm{CHCl}_{3}, c=0.005\right) ; \mathrm{mp}$ $54-56{ }^{\circ} \mathrm{C}$.

\section{$(+)-(S, S)$-Grenadamide (+)-1}

Procedure as for $( \pm)$-grenadamide synthesis from $(+)-\mathbf{2}(308 \mathrm{mg}$, $83 \%)$ as a colourless solid. $[\alpha]_{\mathrm{D}}^{20.5}+12.0^{\circ}\left(\mathrm{CHCl}_{3}, c=0.005\right) ; \mathrm{mp}$ $55-57{ }^{\circ} \mathrm{C}$.

\section{References}

1 N. Sitachitta and W. Gerwick, J. Nat. Prod., 1998, 61, 681.

2 O. Motl, M. Amin and P. Sedmera, Phytochemistry, 1972, 11, 407; S. Wilson and K. Prodan, Tetrahedron Lett., 1976, 47, 4231.

3 J. Al Dulayymi, M. Baird and K. Jones, Tetrahedron, 2004, 60, 341.

4 (a) T. Avery, B. Greatrex, D. Taylor and E. Tiekink, J. Chem. Soc., Perkin Trans. 1, 2000, 1319; (b) T. Avery, G. Fallon, B. Greatrex, S. Pyke, D. Taylor and E. Tiekink, J. Org. Chem., 2001, 66, 7955; (c) T. Avery, D. Taylor and E. Tiekink, J. Org. Chem., 2000, 65, 5531.

5 B. Greatrex and D. Taylor, J. Org. Chem., 2005, 70, 470.

6 S. Chandrasekaran, A. Kluge and J. Edwards, J. Org. Chem., 1977, 42, 3972.

7 D. Barton, D. Crich and W. Motherwell, Tetrahedron, 1985, 41, 3901.

8 C. Guibourdenche, J. Podlech and D. Seebach, Liebigs Ann., 1996, 1121.

9 S. Neset, H. Hope and K. Undheim, Tetrahedron, 1997, 53, 10459.

10 V. Lee and M. Newman, Org. Synth., Coll Vol. VI, 1988, 613.

11 M. Arisawa and M. Yamaguchi, J. Am. Chem. Soc., 2000, 122, 2387.

12 T. de Boer and H. Backer, Org. Synth., Coll Vol. IV, 1963, 250. 